\title{
Discovery of a Perseus-like cloud in the early Universe
}

\section{$\mathrm{H}$ I-to- $\mathrm{H}_{2}$ transition, carbon monoxide and small dust grains at $\mathrm{z}_{\mathrm{abs}} \approx 2.53$ towards the quasar $\mathrm{J0000}+0048^{\star}$}

\author{
P. Noterdaeme ${ }^{1}$, J.-K. Krogager ${ }^{1,2}$, S. Balashev ${ }^{3}$, J. Ge ${ }^{4}$, N. Gupta ${ }^{5}$, T. Krühler ${ }^{6}$, C. Ledoux ${ }^{7}$, M. T. Murphy ${ }^{8}$, \\ I. Pâris ${ }^{9}$, P. Petitjean ${ }^{1}$, H. Rahmani ${ }^{9}$, R. Srianand ${ }^{5}$, and W. Ubachs ${ }^{10}$ \\ 1 Institut d'Astrophysique de Paris, CNRS-UPMC, UMR 7095, 98bis boulevard Arago, 75014 Paris, France \\ e-mail: noterdaeme@iap.fr \\ 2 Dark Cosmology Centre, Niels Bohr Institute, University of Copenhagen, Juliane Maries Vej 30, \\ 2100 Copenhagen $\varnothing$, Denmark \\ 3 Ioffe Physical-Technical Institute of RAS, Polyteknicheskaya 26, 194021 Saint-Petersburg, Russia \\ 4 Department of Astronomy, University of Florida, 211 Bryant Space Science Center, Gainesville, 32611, USA \\ 5 Inter-University Centre for Astronomy and Astrophysics, Post Bag 4, Ganeshkhind, 411007 Pune, India \\ 6 Max-Planck-Institut für extraterrestrische Physik, Giessenbachstraße, 85748 Garching, Germany \\ 7 European Southern Observatory, Alonso de Córdova 3107, Vitacura, Casilla 19001, Santiago 19, Chile \\ 8 Centre for Astrophysics and Supercomputing, Swinburne University of Technology, Melbourne, 3122 Victoria, Australia \\ 9 Laboratoire d'Astrophysique de Marseille, CNRS/Aix Marseille Université, UMR 7326, 13388 Marseille, France \\ 10 Department of Physics and Astronomy, LaserLaB, Vrije Universiteit, De Boelelaan 1081, 1081 HV Amsterdam, \\ The Netherlands
}

Received 23 June 2016 / Accepted 2 September 2016

\begin{abstract}
We present the discovery of a molecular cloud at $z_{\text {abs }} \approx 2.5255$ along the line of sight to the quasar SDSS J 000015.17+004833.3. We use a high-resolution spectrum obtained with the Ultraviolet and Visual Echelle Spectrograph together with a deep multi-wavelength medium-resolution spectrum obtained with X-shooter (both on the Very Large Telescope) to perform a detailed analysis of the absorption lines from ionic, neutral atomic and molecular species in different excitation levels, as well as the broad-band dust extinction. We find that the absorber classifies as a Damped Lyman- $\alpha$ system (DLA) with $\log N(\mathrm{HI})\left(\mathrm{cm}^{-2}\right)=20.8 \pm 0.1$. The DLA has supersolar metallicity $\left(Z \sim 2.5 Z_{\odot}\right.$, albeit to within a factor of two to three) with a depletion pattern typical of cold gas and an overall molecular fraction $f=2 N\left(\mathrm{H}_{2}\right) /\left(2 N\left(\mathrm{H}_{2}\right)+N(\mathrm{HI})\right) \sim 50 \%$. This is the highest $f$-value observed to date in a high- $z$ intervening system. Most of the molecular hydrogen arises from a clearly identified narrow $\left(b \sim 0.7 \mathrm{~km} \mathrm{~s}^{-1}\right)$, cold component in which carbon monoxide molecules are also found, with $\log N(\mathrm{CO}) \approx 15$. With the help of the spectral synthesis code Cloudy, we study the chemical and physical conditions in the cold gas. We find that the line of sight probes the gas deep after the $\mathrm{H}$ I-to- $\mathrm{H}_{2}$ transition in a $\sim 4-5 \mathrm{pc}$-size cloud with volumic density $n_{\mathrm{H}} \sim 80 \mathrm{~cm}^{-3}$ and temperature of only $50 \mathrm{~K}$. Our model suggests that the presence of small dust grains (down to about $0.001 \mu \mathrm{m})$ and high cosmic ray ionisation rate $\left(\zeta_{\mathrm{H}} \sim\right.$ a few times $\left.10^{-15} \mathrm{~s}^{-1}\right)$ are needed to explain the observed atomic and molecular abundances. The presence of small grains is also in agreement with the observed steep extinction curve that also features a $2175 \AA$ bump. Interestingly, the chemical and physical properties of this cloud are very similar to what is seen in diffuse molecular regions of the nearby Perseus complex, despite the former being observed when the Universe was only 2.5 Gyr old. The high excitation temperature of $\mathrm{CO}$ rotational levels towards $\mathrm{J} 0000+0048$ betrays however the higher temperature of the cosmic microwave background. Using the derived physical conditions, we correct for a small contribution $(0.3 \mathrm{~K})$ of collisional excitation and obtain $T_{\mathrm{CMB}}(z=2.53) \approx 9.6 \mathrm{~K}$, in perfect agreement with the predicted adiabatic cooling of the Universe.
\end{abstract}

Key words. quasars: absorption lines - ISM: clouds - ISM: molecules - dust, extinction - cosmology: observations cosmic background radiation

\section{Introduction}

The formation and evolution of galaxies is strongly dependent on the physical properties of the gas in and around galaxies. Indeed, the gas is the reservoir of baryons from which stars form and at the same time, it integrates the chemical and physical outputs from star-formation activity. The gas that is accreted onto galaxies has to cool down and go through different transitional

\footnotetext{
$\star$ Based on observations collected at the European Organisation for Astronomical Research in the Southern Hemisphere under ESO programmes 093.A-0126(A), 096.A-0354(A) and 096.A-0924(B).
}

processes that will determine its properties during its evolution before the final collapse that give birth to stars. Different phases are indeed identified in the interstellar medium, depending on the temperature and density and whether the matter is ionised or neutral (atomic or molecular). In their two-phase model, Field et al. (1969) showed that thermal equilibrium leads neutral gas to segregate into a dense phase, the cold neutral medium (CNM), embedded into a diffuse intercloud phase, the warm neutral medium (WNM). Detailed theoretical and numerical works (e.g. Krumholz et al. 2009; Sternberg et al. 2014) show that a 
transition from $\mathrm{H}$ I to $\mathrm{H}_{2}$ then occurs in the former phase, depending on the balance between $\mathrm{H}_{2}$ formation on the surface of dust grains (e.g. Jura 1974b), and its dissociation by UV photons (e.g. Dalgarno \& Stephens 1970), itself dependent on both selfand dust-shielding.

Observationally, UV absorption spectroscopy of Galactic clouds towards nearby stars reveal that the molecular fraction, $f=2 \mathrm{H}_{2} /\left(2 \mathrm{H}_{2}+\mathrm{HI}\right)$, sharply increases above a $\mathrm{HI}$ column density threshold of $5 \times 10^{20} \mathrm{~cm}^{-2}$. A similar threshold has been found by Reach et al. (1994) from far-infrared emission studies of interstellar clouds, using dust as a tracer for $\mathrm{H}_{2}$. Higher column-density thresholds were observed in the Magellanic Clouds (Tumlinson et al. 2002), which could be the consequence of a higher UV radiation field together with a lower metallicity in these environments. However, it is also possible that a significant fraction of the observed H I column density is actually unrelated to the atomic envelopes of the $\mathrm{H}_{2}$-absorbing clouds (Welty et al. 2012), since $N(\mathrm{HI})$ is derived through unresolved $21-\mathrm{cm}$ emission, while $N\left(\mathrm{H}_{2}\right)$ is measured in absorption.

This highlights the main difficulty in observing the transition regions: because molecular clouds have sizes of only a few tens to a few hundred parsec (e.g. Fukui \& Kawamura 2010) it is very difficult to compare $\mathrm{H}_{2}$ with its associated $\mathrm{HI}$ in the cloud envelope without also integrating nearby atomic gas. High spatial resolution (sub-pc) studies exist for nearby molecular clouds such as the Perseus cloud. Lee et al. (2012) observe relatively uniform H I surface density of $\Sigma_{\mathrm{HI}} \sim 6-8 M_{\odot} \mathrm{pc}^{-2}$ around $\mathrm{H}_{2}$ clouds, in agreement with the theoretical expectations based on $\mathrm{H}_{2}$ microphysics at solar metallicity, assuming CNM a priori (Krumholz et al. 2009) or not (Bialy et al. 2015).

Ideally, we would also like to study the atomic to molecular transition and the subsequent star formation over parsec scales in other galaxies. Observations of nearby galaxies have been possible at slightly sub-kpc resolution, revealing a saturation value around $\Sigma_{\mathrm{HI}} \approx 9 M_{\odot} \mathrm{pc}^{-2}$ (Bigiel et al. 2008). However, the observational techniques applied in the local Universe are not applicable yet in the distant Universe without a further strong loss of spatial resolution. Prescriptions of star-formation over galactic scales, such as the empirical relation between the molecular to atomic ratio and the hydrostatic pressure (e.g. Blitz \& Rosolowsky 2006) are nevertheless available and can be used in evolution models of galaxies (e.g. Lagos et al. 2011), although this corresponds to an extrapolation at high redshift of a phenomenon observed in the local Universe. The increase of sensitivity in sub-mm astromomy has also permited tremendous progress in recent years with detailed studies of the relation between molecular content and star formation at intermediate redshifts (e.g. Tacconi et al. 2013), although still limited to relatively bright and massive galaxies. In addition, observations of atomic gas through $\mathrm{H}$ I $21-\mathrm{cm}$ emission (currently limited to $z<0.4$, e.g. Catinella et al. 2008; Freudling et al. 2011; Fernández et al. 2016) will have to await future radio facilities such as the Square Kilometre Array.

At high redshift, information about gas in the Universe can be accurately obtained through absorption studies towards bright background sources. In particular, damped Lyman- $\alpha$ systems (DLAs, see Wolfe et al. 2005, for a review), with $N(\mathrm{HI}) \geq$ $2 \times 10^{20} \mathrm{~cm}^{-2}$, trace the neutral gas in a cross-section weighted manner, independently of the luminosity of the associated object. DLAs have been conjectured to be originating from gas associated with galaxies, in particular since DLAs contain the bulk of the neutral gas at high redshift (e.g. Prochaska et al. 2005; Prochaska \& Wolfe 2009; Noterdaeme et al. 2009b, 2012a) and their metallicity is increasing with decreasing redshift (e.g.
Rao et al. 2006; Rafelski et al. 2012). While the dust production in the bulk of DLAs seems to be very low (Murphy \& Bernet 2016), the excitation of atomic and molecular species indicates some ongoing star-formation activity (e.g. Wolfe et al. 2004; Srianand et al. 2005; Neeleman et al. 2015). This is also suggested by numerical simulations (e.g. Cen 2012; Bird et al. 2014) or semi-analytical models (e.g. Berry et al. 2016) but direct associations with galaxies remain difficult to establish, with only a few associations between intervening DLAs and galaxies revealed so far at $z>2$ (Møller \& Warren 1993; Møller et al. 2004; Fynbo et al. 2010; Krogager et al. 2012; Noterdaeme et al. 2012b; Bouché et al. 2013; Kashikawa et al. 2014; Hartoog et al. 2015; Srianand et al. 2016). Indeed, statistical studies show a low level of in-situ star formation (Rahmani et al. 2010; Fumagalli et al. 2015), although Ly$\alpha$ emission has been detected through stacking in sub-samples with the highest H I column densities (Noterdaeme et al. 2014), suggesting the latter arise more likely from gas associated with galaxies at small impact parameters.

Noterdaeme et al. (2015a) suggest that $\mathrm{H}_{2}$ is more frequently found in high column density DLAs, but that the measured overall molecular fraction remains much lower than what would be expected from single clouds, even at the typically low metallicities of DLAs. This indicates that most of the observed H I column density along the line of sight is actually unrelated to the $\mathrm{H}_{2}$ core and does not participate in its shielding (see also Noterdaeme et al. 2015b). This again marks the difficulty of distinguishing the $\mathrm{H}$ I envelope of molecular clouds from unrelated atomic gas along the same line of sight. Several methods have been developed to statistically derive the CNM fraction in DLAs. The low detection rate of $21-\mathrm{cm}$ absorption in DLAs indicates high average spin temperatures and hence points to the fact that most DLAs are dominated by WNM (e.g. Kanekar et al. 2014). Neeleman et al. (2015) recently suggest that the bulk of neutral gas could be in the CNM for at least $5 \%$ of DLAs, based on the fine-structure excitation of singly ionised carbon and silicon. This further indicates that such clouds can be as small as a few parsecs. A small size of CNM clouds is also inferred from the lack of correspondence between $21-\mathrm{cm}$ and $\mathrm{H}_{2}$ absorption seen in DLAs (Srianand et al. 2012) and by the partial coverage of the background quasar's broad line region by $\mathrm{H}_{2}$-bearing clouds (e.g. Balashev et al. 2011).

Because $\mathrm{H}_{2}$-bearing systems are rare among the overall DLA population (e.g. Ledoux et al. 2003; Noterdaeme et al. 2008; Jorgenson et al. 2014), directly targeting $\mathrm{H}_{2}$ (instead of blindly targeting $\mathrm{H}$ I gas) could provide a more efficient way to study the phase transition. Unfortunately, $\mathrm{H}_{2}$ lines are located in the Ly $\alpha$ forest and are difficult to detect at low spectral resolution (except when the absorption is in the damped regime, Balashev et al. 2014). In turn, neutral carbon provides an excellent tracer of $\mathrm{H}_{2}$ molecules (e.g. Snow \& McCall 2006), since the ionisation energy of $\mathrm{C} \mathrm{I}$ is close to that of $\mathrm{H}_{2}$ photodissociation. Furthermore, several transitions are located out of the Ly $\alpha$ forest, making it possible to search for strong $\mathrm{C} I$ absorption even at low spectral resolution (see Ledoux et al. 2015). Such selection has led to the first detections of $\mathrm{CO}$ molecules in absorption at $z>1.6$, which also opens the exciting possibility to directly measure the cosmic microwave background (CMB) temperature through the excitation of CO (Noterdaeme et al. 2011). In the two high redshift cases where $\mathrm{H}_{2}$ lines are also covered, we measured overall molecular fractions of about $25 \%$ (Srianand et al. 2008; Noterdaeme et al. 2010), that is significantly higher than in other $\mathrm{H}_{2}$-bearing DLAs, which generally have $f \sim 1 \%$ or less (Ledoux et al. 2003). 
Table 1. Log of observations.

\begin{tabular}{ccccc}
\hline \hline Programme ID & Setting/Mode & $\begin{array}{c}\text { Slit widths } \\
(\operatorname{arcsec})\end{array}$ & Observing dates & $\begin{array}{c}\text { Exposure time } \\
(\mathrm{s})\end{array}$ \\
\hline & & \multicolumn{3}{c}{ UVES } \\
093.A-0126(A) & $390+564$ & $0.9,0.9$ & Aug. 2014 & $5 \times 4800 \mathrm{~s}$ \\
096.A-0354(A) & $390+564$ & $0.9,0.7$ & Oct.-Nov. 2015 & $6 \times 4200 \mathrm{~s}$ \\
096.A-0354(A) & $437+760$ & $0.9,0.9$ & Nov. 2015 & $2 \times 4200 \mathrm{~s}$ \\
\hline & & \multicolumn{3}{c}{ X-shooter } \\
096.A-0924(B) & Nodding & $1.3,0.9,1.2$ & Sep-Dec 2015, Aug 2016 & $8 \times 2 \times(1400,1430,3 \times 480)$ \\
\hline
\end{tabular}

Notes. The different values for slit width correspond to different arms, ordered by increasing wavelength: BLUE, RED for UVES and UVB, VIS, NIR for $\mathrm{X}$-shooter. The exposure times for $\mathrm{X}$-shooter data are detailled as (number of $\mathrm{OB}) \times($ nodding positions) $\times($ exposure time for a given position), with the NIR being sub-divided in 3 integrations (NDIT).

In our quest for molecular-rich systems in the Sloan Digital Sky Survey-III (see Ledoux et al. 2015, for the corresponding search in the SDSS-II), we found a new case at $z_{\text {abs }} \sim 2.5$ towards the quasar SDSS J000015.17+004833.3 (hereafter J0000+0048) with strong C I absorption and a prominent $2175 \AA$ A bump, which we followed-up with the Very Large Telescope. The characteristics of this system in terms of molecular fraction, $\mathrm{CO}$ column density, and metallicity supersede all values measured in DLAs so far. A cold, molecule-bearing component is clearly identified, allowing us to perform an unprecedentedly detailed analysis of the chemical and physical conditions in the molecular cloud and to study the transition from the atomic to the molecular phase. We present our observations in Sect. 2, the absorption-line analysis of ionic, atomic and molecular species in Sect. 3. We discuss the metallicity and dust abundance in Sect. 4, the extinction in Sect. 5 and the physical conditions in the cloud in Sect. 6. We use $\mathrm{CO}$ to measure the cosmic microwave background temperature at $z=2.53$ in Sect. 7. We search for star-formation activity in Sect. 8 and conclude in Sect. 9.

\section{Observations and data reduction}

\subsection{UVES}

High-resolution spectroscopic observations of J0000+0048 $\left(z_{\mathrm{em}} \approx 3.03\right)$ were carried out using the Ultraviolet and Visual Echelle Spectrograph (UVES; Dekker et al. 2000) mounted on the unit 2 of the $8.2 \mathrm{~m}$ Very Large Telescope (VLT) at Paranal observatory under two distinct ESO programmes 093.A-0126(A) in 2014 (P93) and 096.A-0354(A) in 2015 (P96). The former observations were all performed using the standard beam splitter with 390+564 setting at a slit width of $0.9^{\prime \prime}$.

The latter (P96) were mostly performed with the same setting but with a narrower slit width of $0.7^{\prime \prime}$ in the red arm, and attached Th-Ar calibration. We also observed the quasar $2 \times 4200 \mathrm{~s}$ with central wavelength set to $760 \mathrm{~nm}$ in the red arm in order to extend the spectral coverage over the redshifted absorption position of useful metal species (Zn II, Fe II). These were taken with a $0.9^{\prime \prime}$-wide slit. We used a CCD readout with $2 \times 2$ binning and set the slit position to paralactic angle for all the observations to minimise the effects of atmospheric dispersion. A summary of the observations is shown in Table 1.

The data were reduced using UVES Common Pipeline Library (CPL) data reduction pipeline release 6.5 using an optimal extraction algorithm (Horne 1986). We used 4th order polynomials to find the dispersion solution. The individual science exposures were shifted to the heliocentric-vacuum frame correcting for the observatory's motion towards the line of sight at the exposure mid point, using the air-to-vacuum relation from Ciddor (1996).

All exposures taken with the BLUE arm were obtained using a $0.9^{\prime \prime}$-wide slit and extracted onto a fixed wavelength grid with a pixel step of $2.5 \mathrm{~km} \mathrm{~s}^{-1}$ which corresponds to the pixel size on the CCD. The spectrum of each echelle order was interpolated onto this global grid so that no further rebinning was required neither when merging orders of an exposure nor when combing different exposures. Similarly, exposures taken with the RED arm have higher resolution and smaller pixel sizes and were extracted onto a grid with a pixel step of $2.0 \mathrm{~km} \mathrm{~s}^{-1}$.

Cosmic ray residuals and bad pixels were flagged using a semi-interactive procedure and the data quality was checked to remove a few failed exposures. Individual 1D extractions were then scaled and combined together into three final 1D spectra: a "blue" spectrum, with spectral resolution $6.30 \mathrm{~km} \mathrm{~s}^{-1}$; a "red" spectrum with resolution ranging from 5.45 to $5.80 \mathrm{~km} \mathrm{~s}^{-1}$ corresponding to all exposures taken with $0.9^{\prime \prime}$ slit and a higher resolution "red" spectrum, with resolution $4.60-4.70 \mathrm{~km} \mathrm{~s}^{-1}$, combining the $0.7^{\prime \prime}$-wide slit exposures. The average $\mathrm{S} / \mathrm{N}$ per pixel is about 8 at $4000 \AA$ in the blue spectrum. The combined red spectrum has in turn $S / N \sim 20$ at $5300 \AA$.

\subsection{X-shooter}

Deep, medium-resolution spectroscopic observations of $\mathrm{J} 0000+0048$ over the full wavelength range from $\sim 320 \mathrm{~nm}$ to $\sim 2.25 \mu \mathrm{m}$ were carried out at the VLT unit 3 using X-shooter under ESO programme 096.A-0924(B). We performed all observations using the Nodding mode and slit widths of 1.3", $0.9^{\prime \prime}$ and $1.2^{\prime \prime}$ for the UVB, VIS and NIR arm, respectively, and a binning of $1 \times 2$. We used different slit position angles to maximise the spatial coverage around the quasar location. The $\log$ of observations is shown in Table 1.

Our X-shooter data reduction heavily relied on the pipeline supplied by ESO in its version 2.5.2 (Modigliani et al. 2010). For every position angle, we used the pipeline to apply a flat-field correction, order tracing and rectification of individual frames in each nodding position individually. In the UVB and VIS arms, the sky spectrum was subtracted using regions in the $11^{\prime \prime}$-long $\mathrm{X}$-shooter slit free of signal. In the NIR, the intensity of the sky spectrum is high so we used the frame taken at the alternate nodding position closest in time for background subtraction. Wavelength and flux calibrations were performed using arc lamp lines observed during daytime and the nightly spectrophotometric standard, respectively. 


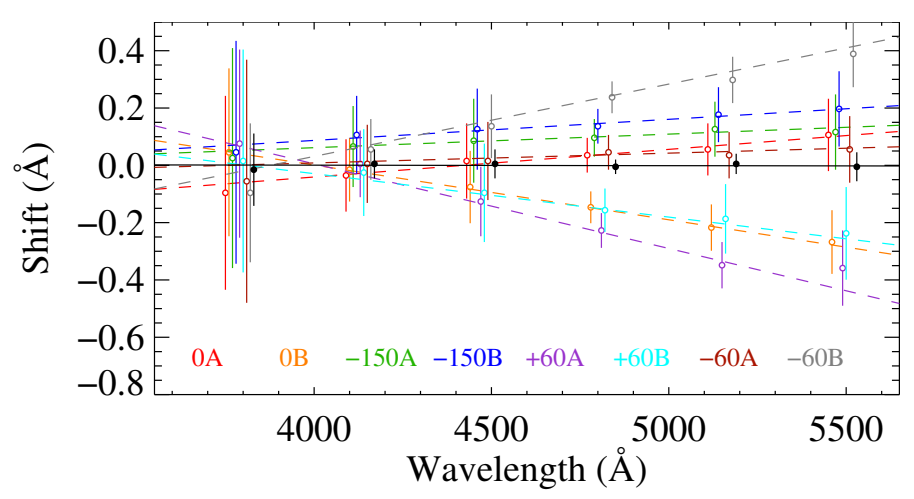

Fig. 1. X-shooter wavelength distortion in the UVB arm for the different exposures. Points with different colours represent averages in $200 \AA$ chunks for different PAs (whose names, given in the bottom, correspond to the angle in degrees east of north, and " $\mathrm{A} / \mathrm{B}$ " for first/second observation with same PA). The black filled dots correspond to the combined spectrum.

This process provided us with sky-subtracted, wavelengthand flux-calibrated 2D spectra. After cosmic-ray and bad-pixel detection using our own algorithm based on Laplacian-filter edge detection, we averaged these frames with variance weighting. This yielded a single frame per arm and position angle. We then obtained the 1D spectra by optimal extraction, where the appropriate weights along the spatial direction were derived using a Moffat-function fitted to the data. Finally, the spectra were corrected for Galactic foreground reddening (Schlafly \& Finkbeiner 2011) and converted into a vacuum heliocentric reference frame. We checked the quality of the flux calibration by comparing the spectra against each other and found agreement within $15 \%$ in the UVB (and better in the other arms). Similarly, we found no evidence for significant chromatic slit losses in the data by comparing with accurate multi-band photometry (see Sect. 5).

Because X-shooter sits at the Cassegrain focus of the VLT, shifts in the wavelength solution by about $0.5 \AA$ are not uncommon due to flexure (e.g. Bristow et al. 2011). This is particularly expected when observations are performed off the parallactic angle. Thanks to UVES observations of the same object, we indeed noticed and corrected for wavelength distortions in the UVB arm: we smoothed the UVES spectrum to X-shooter's spectral resolution and cross-correlated the resulting spectrum with the X-shooter data over $200 \AA$ chunks. The wavelength distortions were different for each observation but could be very well approximated by a linear function of wavelength (see also Chen et al. 2014). We thus corrected for this distortion before combining the individual 1D exposures. This led to an improvement of the wavelength calibration accuracy by a factor of more than ten compared to the pipeline results (Fig. 1) without losing spectral resolution in the final spectrum due to blurring effect. The combined $\mathrm{X}$-shooter spectrum had a $\mathrm{S} / \mathrm{N}$ ratio $\sim 35,55$ and 25 and resolution around 75, 32 and $35 \mathrm{~km} \mathrm{~s}^{-1}$ in the UV, visual and near infrared, respectively.

\section{Absorption line analysis}

We detect metal absorption lines in the $z_{\mathrm{abs}}=2.525$ absorption system from various ionisation stages from high-ionisation species (e.g. C IV, Al III, see Fig. 2) and singly ionised species (Si II, Ni II, Zn II and Fe II) spread over roughly $400 \mathrm{~km} \mathrm{~s}^{-1}$. We detect a narrow component at the extreme red edge of the profile
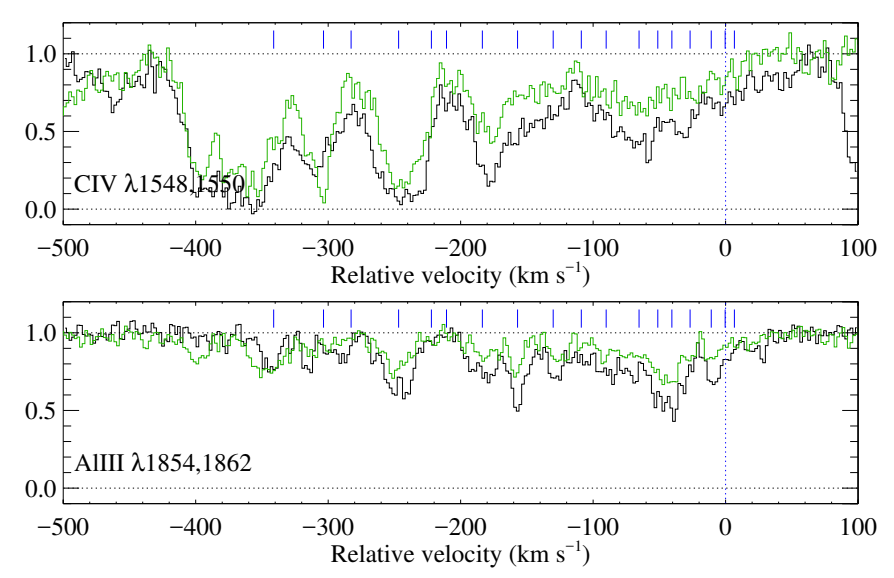

Fig. 2. High ionisation species detected towards J0000+0048 in the UVES spectrum. The green profile corresponds the reddest transition for each doublet. The ticks marks correspond to the location of singly ionised metal components, and the zero-velocity scale is set at the position of the molecular component. In this, and in all analoguous figures presenting absorption lines, the $y$ axis shows the normalised flux.

in which we also detect neutral ( $\mathrm{S} \mathrm{I}, \mathrm{Mg} \mathrm{I}, \mathrm{Cl} \mathrm{I}, \mathrm{CI}$ ) and molecular $\left(\mathrm{H}_{2}, \mathrm{CO}\right)$ species. While the overall kinematic profile is interesting - with a large velocity extent and absorption strengths varying differently with velocity for different ions, suggesting galactic winds - we mostly focus on the molecular component in this paper. We used VPFIT (Carswell \& Webb 2014) version 10.3 to model the absorption profiles using multi-component Voigtprofile fitting in order to obtain redshifts, Doppler parameters and column densities of different species.

During our analysis, we combined the two red UVES spectra into a single spectrum using an inverse variance weighting. In principle, the resulting spectral point spread function (SPSF) becomes the combination of the two Gaussian SPSF as done by Carswell et al. (2012). In practice, because we are using data from the same instrument with resolutions that differ by only $20 \%$, the resulting SPSF can very well be approximated by a single Gaussian with resolution ranging from 5 to $5.25 \mathrm{~km} \mathrm{~s}^{-1}$ over the region covered by both original spectra. We checked that fitting both red spectra simultaneously or using their combination provided consistent results. Therefore we here provide the results using the combined spectrum. For the particular case of $\mathrm{CO}$, we tested this in more detail and also provide the simultaneous fit to the two sets of UVES data.

\subsection{Atomic hydrogen}

We determined the H I column density of the system by simultaneously fitting the continuum of the background quasar together with a Voigt profile to the damped Ly $\alpha$ absorption line. Higherorder Lyman lines were not usable due to blending with stronger damped $\mathrm{H}_{2}$ lines (see Sect. 3.4). We used the X-shooter spectrum since it has much higher signal-to-noise ratio than the UVES spectrum in this region. Moreover the X-shooter spectrum is flux calibrated, making it easier to determine the continuum placement. We obtained $\log N(\mathrm{HI})=20.8 \pm 0.1$. As expected, the UVES data is consistent with this value, see Fig. 3 .

\subsection{Metals}

About twenty velocity components with a wide dynamical range of optical depths can be distinctly identified in the profiles of Si II 


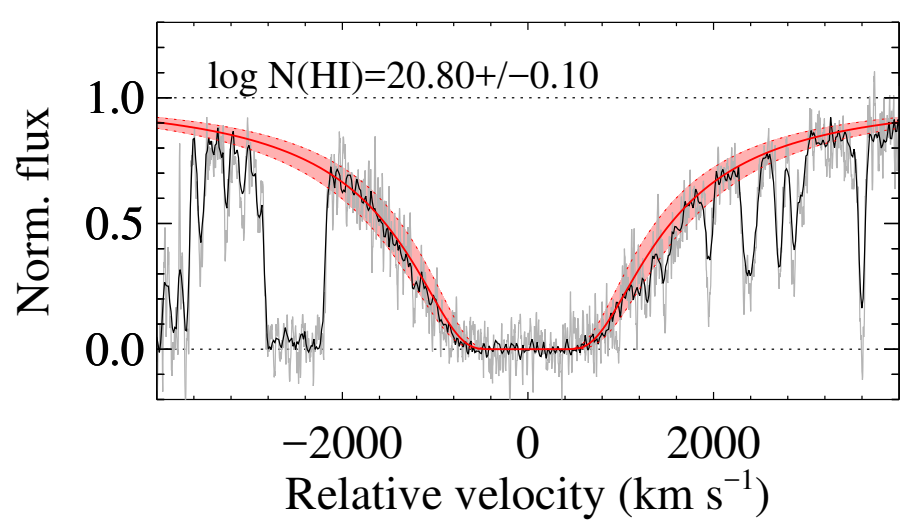

Fig. 3. Measurement of the H I column density at $z_{\mathrm{abs}}=2.525$. The $\mathrm{X}$-shooter data is represented in black, while the UVES data, boxcarsmoothed by 5 pixels is represented in grey. The Voigt profile fit is shown in red with the associated uncertainty as a shaded region.

and Fe II thanks to several transitions spanning a range of oscillator strengths. We used these species together with S I (whose $1807 \AA$ transition is blended with Si II $\lambda 1808$ ) to obtain a first solution for the component structure. We then included Ni II and Fe II and let the column densities vary freely while the Doppler parameters and redshifts were tied together for singly ionised species. The redshift and Doppler parameters for neutral species ( $\mathrm{S}$ I and $\mathrm{Mg} \mathrm{I}$ ) were kept independent.

A very narrow component $\left(b \approx 0.6 \mathrm{~km} \mathrm{~s}^{-1}\right)$ corresponding to the neutral and molecular species is clearly seen at the extreme red edge of the profiles of Si II $\lambda 1808$ and Zn II $\lambda \lambda 2026,2062$ while much weaker in Fe II lines and not detected at all in Ni II (see the component at $v=0 \mathrm{~km} \mathrm{~s}^{-1}$ in Fig. 4). This already indicates a high level of dust depletion since the later species are refractory while zinc is a volatile element (e.g. Pettini et al. 1997). We use this narrow component $(z=2.52546)$ as the reference for the zero-velocity in all figures and discussions in the paper. We also note that we did not make any assumption on the velocity structure (that is, redshift and Doppler parameter) of this component and we fitted the molecular, atomic, and ionic species independently. The results from fitting the lines are shown in Fig. 4 and the corresponding parameters provided in Table 2. We measured total column densities of respectively $\log N\left(\mathrm{~cm}^{-2}\right)=$ $15.93 \pm 0.17$ (Si II), $13.99 \pm 0.04$ (Ni II), 14.09 \pm 0.45 ( $\mathrm{Zn} \mathrm{II})$ and $15.14 \pm 0.03$ (Fe II). We note that the Zn II $\lambda 2062$ line might be blended with $\mathrm{Cr}$ II absorption (Zn II $\lambda 2026$ as well, but the nearby $\mathrm{Cr}$ II line has very low oscillator strength). However, we do not detect the unblended $\mathrm{Cr}$ II $\lambda \lambda 2056,2066$ lines despite their strong oscillator strengths. The effect of chromium on the measurement of $N(\mathrm{Zn}$ II $)$ should therefore be negligible.

We also detect PII $\lambda 1532$ (P II $\lambda 1301$ is unfortunately lost within the saturated $\mathrm{O} I \lambda 1302$ profile) in the two strongest components, although close to the noise level. We therefore fixed the redshifts and Doppler parameters to the values determined from other metals and obtain $\log N(\mathrm{P}$ II $) \sim 14.5 \pm 0.1$. Finally, S II lines are detected but redshifted into the Ly $\alpha$ forest. While two of them $(\lambda \lambda 1250,1253)$ were not severely blended, their oscillator strengths are similar. This, together with the low signal-to-noise ratio achieved in this region of the UVES spectrum prevented us from getting meaningful constraints through line fitting, in particular for the strong narrow component. However, we checked that the data are consistent with the expected profile assuming a solar zinc-to-sulphur ratio in the gas phase.

C II lines are also detected in the UVES spectrum; however, these are saturated and did not provide any meaningful constraint on the column density of ionised carbon. In turn, the $\mathrm{C}$ II $^{*} \lambda 1335$ fine-structure doublet lines are not apparently saturated as can be appreciated from Fig. 5. C II* $\lambda 1037$ is unfortunately completely blended with intervening Ly $\alpha$ forest absorption. Measuring the corresponding column densities therefore remains hazardous due to the many components overlapping in the doublet and the fact that the velocity decomposition differs from that of other metals. In addition, the strongest component is on the nonlinear part of the curve of growth. We obtained $\log N\left(\mathrm{C} \mathrm{II}^{*}\right) \sim 15$ in that component, with $b \sim 0.7 \mathrm{~km} \mathrm{~s}^{-1}$. These values should be considered with great caution as the fit was sensitive to the initial guess, leading to uncertainties larger than an order of magnitude.

\subsection{Neutral carbon}

The strong C I absorption lines that were used to select the system from the low-resolution SDSS spectrum are resolved in our UVES spectrum into different components and different finestructure levels. We detect all three fine-structure levels of neutral carbon's ground state triplet $\left(2 \mathrm{~s}^{2} 2 \mathrm{p}^{2}{ }^{3} \mathrm{P}_{0,1,2}^{e}\right)$ in five components, the strongest of which (by a factor of more than a hundred in column density) is associated with the narrow component seen in both the low-ionisation metal profile and in molecular absorption $\left(\mathrm{H}_{2}\right.$ and $\left.\mathrm{CO}\right)$. We simultaneously fitted all components from the fine-structure levels $\left(J=\{0,1,2\}\right.$, here denoted $\mathrm{CI}, \mathrm{CI}^{*}$, $\mathrm{C} \mathrm{I}^{* *}$, respectively), tying Doppler parameters and redshifts for a given velocity component. We note that while this decreases the number of free parameters, it is based on the reasonable assumption that the fine-structure levels share the same physical origin. We used the lines at $\lambda_{\text {rest }} \approx 1560$ and $1656 \AA$, located outside the Ly $\alpha$ forest, to constrain the fit (see Fig. 6). Including other lines (e.g. C I $\lambda \lambda 1277,1328)$ did not improve the constraints due to the blends, lower $\mathrm{S} / \mathrm{N}$ and lower spectral resolution. The results are provided in Table 3.

\subsection{Molecular hydrogen}

The spectrum of $\mathrm{J} 0000+0048$ is crowded with very strong Lyman $\left(\mathrm{B}^{1} \Sigma_{\mathrm{u}}^{+}\left(v^{\prime}\right)-\mathrm{X}^{1} \Sigma_{g}^{+}(0)\right)$ and Werner $\left(\mathrm{C}^{1} \Pi_{u}\left(v^{\prime}\right)-\mathrm{X}^{1} \Sigma_{g}^{+}(0)\right)$ lines from molecular hydrogen bluewards of $4000 \AA$ (see Fig. 8). Since the X-shooter spectrum has a much higher S/N and an extended wavelength coverage in the blue compared to the UVES spectrum, we used both spectra for the analysis of $\mathrm{H}_{2}$, after normalising the spectra using a spline function. The UVES spectrum was particularly useful to identify regions blended with intervening Ly $\alpha$ absorption from the forest, which were subsequently excluded during the fitting process.

Because the first ionisation potential of carbon, $11.26 \mathrm{eV}$, is very close to that of $\mathrm{H}_{2}$ dissociation, carbon is usually considered a good tracer of molecular hydrogen (e.g. Srianand et al. 2005). While there is no one-to-one correspondence, we can expect $\mathrm{H}_{2}$ to be present in the five components in which $\mathrm{CI}$ is detected. Unfortunately, because the $\mathrm{H}_{2}$ lines are strongly saturated, it was impossible to distinguish the several close components in their profile. We therefore measured only the total $\mathrm{H}_{2}$ column density by modelling the absorption profile using a single velocity component. This model is dominated by the reddest narrow component, for which the $\mathrm{C}$ I column density is about two orders of magnitude higher than in the rest of the components. We tied together the redshifts for the different $\mathrm{H}_{2}$ rotational levels, under the assumption that they arise from the same physical cloud. Absorption lines for the low rotational levels $(J \leq 2)$ are damped, meaning that the column density was well constrained while the 

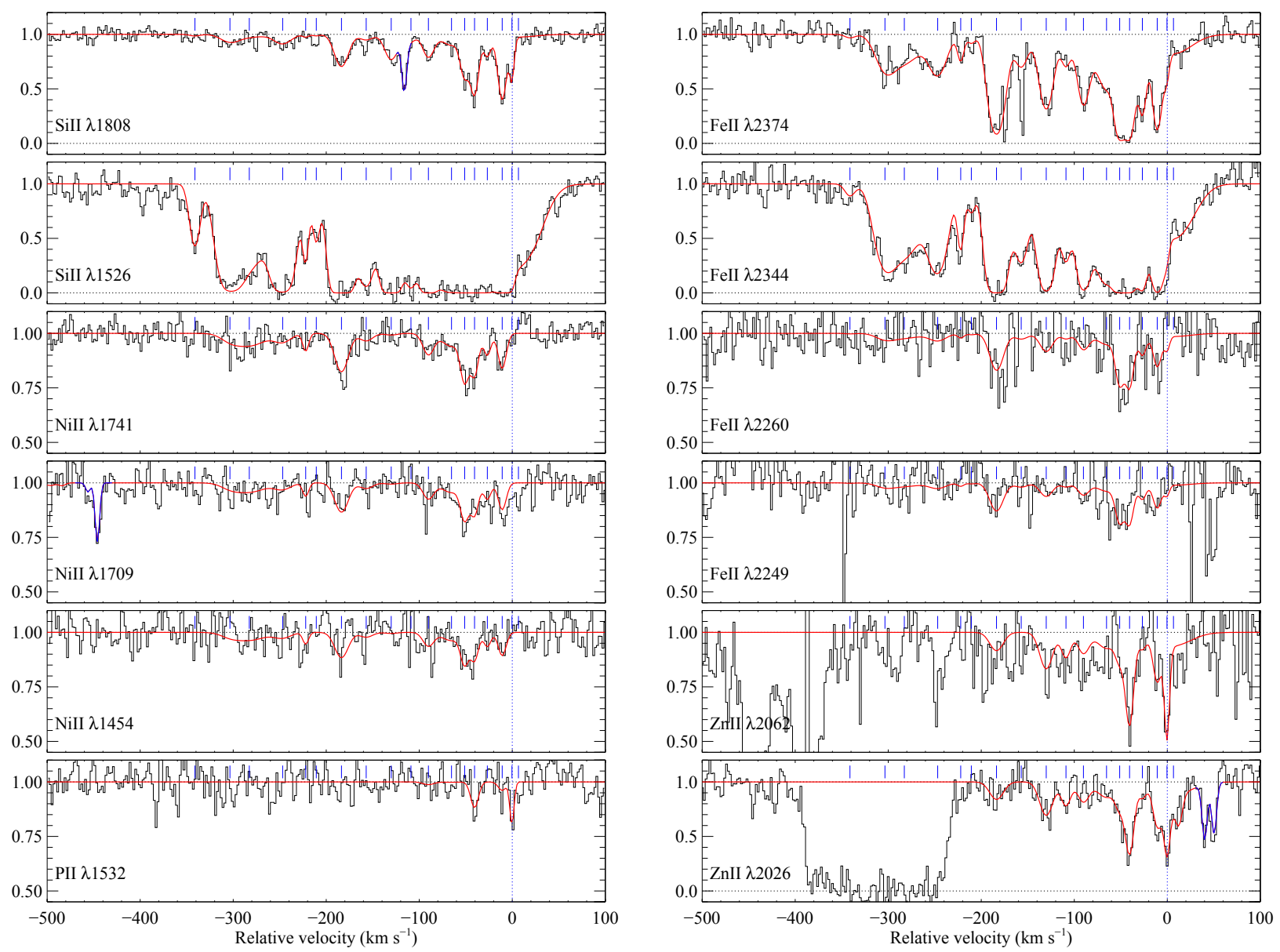

Fig. 4. Multi-component Voigt-profile fit to singly ionised species (red), overlayed on top of the normalised UVES data. Regions marked in blue correspond to species other than the one labelled; for example, the blue region in Si II $\lambda 1808$ is due to absorption by S I $\lambda 1807$.

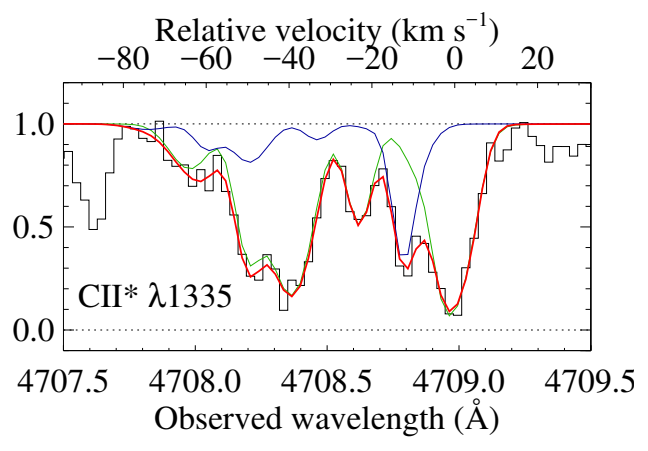

Fig. 5. Fit to the $\mathrm{C} \mathbf{I}^{*} \lambda 1335$ absorption profile (UVES data). The blue and green curves correspond to the contribution from each transition of the doublet. The top axis shows the relative velocity corresponding to the strongest transition (green, with $\lambda=1335.7077$ A rest-frame).

profile does not directly depend on (and therefore did not constrain) the Doppler parameter.

Figure 7 shows the excitation diagram of $\mathrm{H}_{2}$, which presents the population in each rotational level against the energy of that level:

$\frac{N\left(\mathrm{H}_{2}, J^{\prime}\right)}{g\left(\mathrm{H}_{2}, J^{\prime}\right)}=\frac{N\left(\mathrm{H}_{2}, J\right)}{g\left(\mathrm{H}_{2}, J\right)} \mathrm{e}^{-E_{J J^{\prime}} / k T_{J J^{\prime}}}$,

where $E_{J J^{\prime}}$ is the energy difference between levels $J$ and $J^{\prime}$, $g\left(\mathrm{H}_{2}, J\right), g\left(\mathrm{H}_{2}, J^{\prime}\right)$ are the respective spin statistical weights and $T_{J J^{\prime}}$ is the excitation temperature. $T_{01}$ is generally considered as
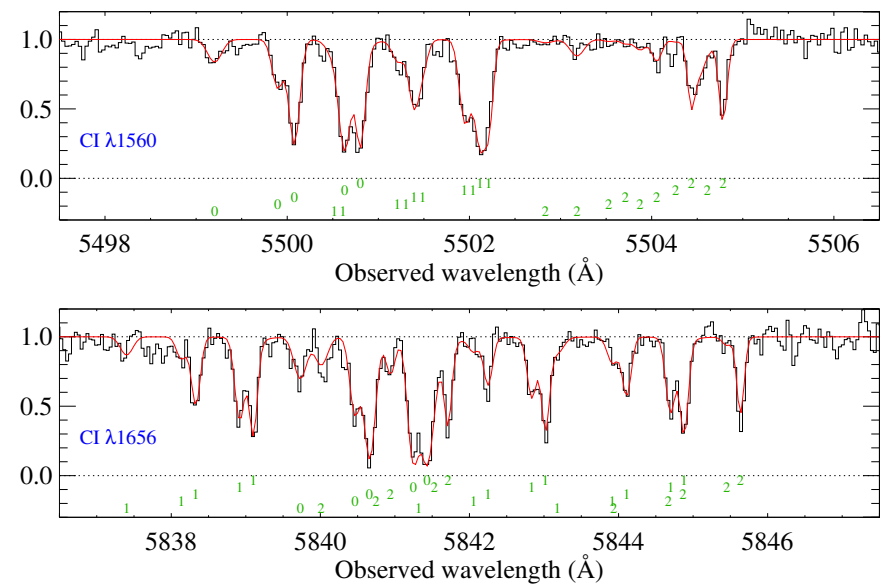

Fig. 6. Fit to the neutral carbon lines (from UVES data). The green numbers below each plot indicate the fine structure level $J$ for each of the five detected $\mathrm{C}$ I components.

a very good indicator of the kinetic temperature of the gas at such high column density, where selective self-shielding is no longer at play and the low rotational levels are easily thermalised thanks to short collisional time-scales (Roy et al. 2006; Le Petit et al. 2006). In turn, the high rotational levels are characterised by a higher excitation temperature. This is expected and seen in interstellar clouds because of the very slow infrared relaxation after UV or formation pumping into high- $J$ levels. Moreover 
Table 2. Result of Voigt profile fitting to singly ionised metal lines.

\begin{tabular}{ccccccc}
\hline \hline$z_{\text {abs }}$ & $v\left(\mathrm{~km} \mathrm{~s}^{-1}\right)$ & $b\left(\mathrm{~km} \mathrm{~s}^{-1}\right)$ & $\log N(\mathrm{SiII})$ & $\log N(\mathrm{NiII})$ & $\log N(\mathrm{ZnII})$ & $\log N(\mathrm{FeII})$ \\
\hline 2.521448 & -341 & $6.64 \pm 0.91$ & $13.31 \pm 0.04$ & & & $12.34 \pm 0.08$ \\
2.521891 & -303 & $13.26 \pm 1.61$ & $14.21 \pm 0.08$ & $12.47 \pm 0.54$ & & $13.59 \pm 0.19$ \\
2.522135 & -283 & $22.62 \pm 4.51$ & $14.03 \pm 0.15$ & $13.08 \pm 0.16$ & & $13.79 \pm 0.13$ \\
2.522557 & -247 & $12.26 \pm 0.68$ & $14.31 \pm 0.05$ & $12.65 \pm 0.20$ & & $13.72 \pm 0.03$ \\
2.522848 & -222 & $3.02 \pm 0.44$ & $13.28 \pm 0.07$ & $12.54 \pm 0.15$ & & $13.08 \pm 0.04$ \\
2.522982 & -211 & $4.49 \pm 0.98$ & $13.14 \pm 0.06$ & & & $12.64 \pm 0.06$ \\
2.523301 & -184 & $9.52 \pm 0.28$ & $14.80 \pm 0.03$ & $13.25 \pm 0.05$ & $12.07 \pm 0.15$ & $14.36 \pm 0.03$ \\
2.523613 & -157 & $9.16 \pm 1.08$ & $13.96 \pm 0.05$ & $12.49 \pm 0.24$ & & $13.50 \pm 0.04$ \\
2.523930 & -130 & $8.89 \pm 0.49$ & $14.64 \pm 0.04$ & $11.81 \pm 1.09$ & $12.36 \pm 0.07$ & $14.00 \pm 0.02$ \\
2.524179 & -109 & $6.08 \pm 1.25$ & $13.84 \pm 0.10$ & & $12.06 \pm 0.12$ & $13.34 \pm 0.06$ \\
2.524400 & -90 & $7.58 \pm 0.92$ & $14.53 \pm 0.07$ & $12.88 \pm 0.10$ & $12.04 \pm 0.14$ & $13.89 \pm 0.05$ \\
2.524692 & -65 & $13.37 \pm 6.16$ & $14.41 \pm 0.24$ & $12.88 \pm 0.25$ & $12.14 \pm 0.23$ & $13.94 \pm 0.21$ \\
2.524858 & -51 & $5.25 \pm 1.32$ & $14.72 \pm 0.14$ & $13.12 \pm 0.14$ & $12.01 \pm 0.31$ & $14.29 \pm 0.13$ \\
2.524983 & -41 & $4.93 \pm 0.77$ & $14.93 \pm 0.06$ & $13.05 \pm 0.10$ & $12.65 \pm 0.06$ & $14.31 \pm 0.09$ \\
2.525145 & -27 & $4.31 \pm 0.76$ & $14.33 \pm 0.09$ & $12.71 \pm 0.12$ & $11.70 \pm 0.26$ & $13.85 \pm 0.05$ \\
2.525333 & -11 & $5.06 \pm 0.43$ & $14.99 \pm 0.03$ & $12.98 \pm 0.07$ & $12.25 \pm 0.12$ & $14.08 \pm 0.05$ \\
2.525456 & 0 & $0.59 \pm 0.11$ & $15.55 \pm 0.41$ & & $14.03 \pm 0.52$ & $13.51 \pm 0.43$ \\
2.525538 & +7 & $26.22 \pm 2.94$ & $14.06 \pm 0.08$ & & $12.33 \pm 0.25$ & $13.66 \pm 0.07$ \\
Total & & & $15.93 \pm 0.17$ & $13.99 \pm 0.04$ & $14.09 \pm 0.45$ & $15.14 \pm 0.03$ \\
\hline
\end{tabular}

Table 3. Column density of neutral carbon in differemt fine-structure levels.

\begin{tabular}{ccccc}
\hline \hline$z_{\text {abs }}$ & $b\left(\mathrm{~km} \mathrm{~s}^{-1}\right)$ & $\log N(\mathrm{C} \mathrm{I}, J=0)$ & $\log N(\mathrm{C} \mathrm{I}, J=1)$ & $\log N(\mathrm{C} \mathrm{I}, J=2)$ \\
\hline 2.524432 & $4.96 \pm 0.71$ & $12.76 \pm 0.05$ & $12.74 \pm 0.11$ & $12.69 \pm 0.07$ \\
2.524876 & $3.41 \pm 0.67$ & $13.05 \pm 0.04$ & $12.73 \pm 0.07$ & $12.38 \pm 0.17$ \\
2.524993 & $2.57 \pm 0.32$ & $13.61 \pm 0.07$ & $13.31 \pm 0.03$ & $12.67 \pm 0.07$ \\
2.525347 & $2.84 \pm 0.26$ & $13.65 \pm 0.06$ & $13.46 \pm 0.02$ & $12.46 \pm 0.15$ \\
2.525458 & $0.81 \pm 0.04$ & $16.10 \pm 0.08$ & $15.54 \pm 0.14$ & $14.67 \pm 0.11$ \\
\hline
\end{tabular}

collisional de-excitation becomes difficult at the high- $J$ levels, where the level spacing becomes so large (several hundred $\mathrm{cm}^{-1}$ ) that these amounts of energy cannot be transferred in collisions, particularly at the densities and temperatures seen in the ISM. This leads to the observed non-Boltzmann distribution. We also note that the observed excitation diagram corresponds to integrated values and that possible additional warmer components with lower $N\left(\mathrm{H}_{2}\right)$ will mostly contribute to the high- $J$ levels.

We measured $T_{01}=51 \pm 2 \mathrm{~K}$, which is lower than the value typically seen in $\mathrm{H}_{2}$-bearing DLAs (Srianand et al. 2005, $T \sim 150 \mathrm{~K}$ ), and closer to what is seen in our Galaxy, with an average of about $77 \mathrm{~K}$ (Rachford et al. 2002). The kinetic temperature $T_{\text {kin }} \approx T_{01}$ corresponds to a Doppler parameter for $\mathrm{H}_{2}$ of $b_{\text {th }} \approx 0.65 \mathrm{~km} \mathrm{~s}^{-1}$ if we assume thermal broadening only. However, because turbulent broadening is also likely present, as indicated by similar Doppler parameters for much heavier species, this value should be considered as a lower limit to the line broadening. We were able to get a more realistic estimate of the Doppler parameter by quadratically adding this pure thermal value to the turbulent $b$-value seen for heavier species (for which $b_{\text {th }}$ is negligible, see Fig. 16) and obtained $b=\sqrt{b_{\mathrm{th}}^{2}+b_{\mathrm{turb}}^{2}} \approx 1.0 \mathrm{~km} \mathrm{~s}^{-1}$.

However, it has been observed in several $\mathrm{H}_{2}$-bearing systems that the Doppler parameter can be an increasing function of the rotational level (e.g. Lacour et al. 2005; Noterdaeme et al. 2007; Albornoz Vásquez et al. 2014), possibly due to more turbulent an warmer external layers where UV pumping of $\mathrm{H}_{2}$ is enhanced (Balashev et al. 2009). We therefore also performed a fit using a high $b$-value of $5 \mathrm{~km} \mathrm{~s}^{-1}$. The resulting parameters are provided
Table 4. Column density in different rotational levels of $\mathrm{H}_{2}$ for the three values of the Doppler parameter.

\begin{tabular}{cccc}
\hline \hline $\begin{array}{c}\text { Rot. level } \\
(J)\end{array}$ & $\begin{array}{c}b=1.0 \\
\left(\mathrm{~km} \mathrm{~s}^{-1}\right)\end{array}$ & $\begin{array}{c}\log N\left(\mathrm{H}_{2}, J\right) \\
b=0.65 \\
\left(\mathrm{~km} \mathrm{~s}^{-1}\right)\end{array}$ & $\begin{array}{c}b=5 \\
\left(\mathrm{~km} \mathrm{~s}^{-1}\right)\end{array}$ \\
\hline 0 & $20.29 \pm 0.02$ & $20.29 \pm 0.02$ & $20.31 \pm 0.02$ \\
1 & $19.81 \pm 0.02$ & $19.80 \pm 0.02$ & $19.79 \pm 0.02$ \\
2 & $18.77 \pm 0.03$ & $18.77 \pm 0.03$ & $18.70 \pm 0.03$ \\
3 & $18.67 \pm 0.02$ & $18.67 \pm 0.02$ & $18.57 \pm 0.03$ \\
4 & $17.32 \pm 0.12$ & $17.37 \pm 0.11$ & $15.08 \pm 0.10$ \\
5 & $14.50 \pm 0.36$ & $14.79 \pm 0.62$ & $14.42 \pm 0.16$ \\
\hline Total & $20.43 \pm 0.02$ & $20.43 \pm 0.02$ & $20.44 \pm 0.02$ \\
\hline
\end{tabular}

in Table 4. We obtained a total column density of $\log N\left(\mathrm{H}_{2}\right)=$ $20.43 \pm 0.02$, which implies an overall molecular fraction of $f=$ $2 N\left(\mathrm{H}_{2}\right) /\left(2 N\left(\mathrm{H}_{2}\right)+N(\mathrm{HI})\right)=0.46 \pm 0.07$, that is the highest value measured to date in a quasar-DLA. This also corresponds to a strict lower limit to the molecular fraction in the cold component.

\subsection{Deuterated molecular hydrogen}

Several lines of deuterated molecular hydrogen are also detected in both the UVES and X-shooter spectra. However, HD lines are often blended with Ly $\alpha$ forest or $\mathrm{H}_{2}$ absorption and are saturated in the low $\mathrm{S} / \mathrm{N}$ ratio UVES spectrum but weak in the medium resolution $\mathrm{X}$-shooter spectrum. We therefore used a different 


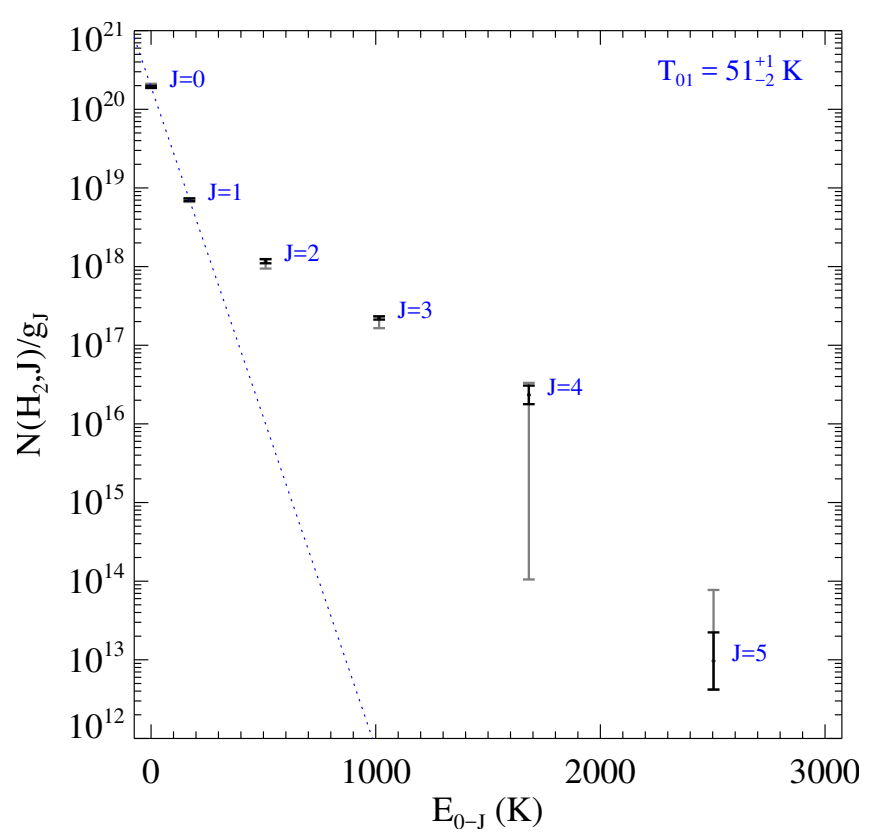

Fig. 7. $\mathrm{H}_{2}$ excitation diagram. The black points and error bars correspond to the fit with $b=1.0 \mathrm{~km} \mathrm{~s}^{-1}$. Grey error bars correspond to extrema with $b=0.65$ and $5 \mathrm{~km} \mathrm{~s}^{-1}$.

fitting procedure based on a Markov chain Monte Carlo method. We considered L0R0, L4R0, L5R0, L6R0, L8R0 and W0R0, locally re-normalised, as well as L11R0 and L14R0 (covered only by X-shooter). We used two components with fixed redshifts $(z=2.525458$ and $z=2.525348)$ corresponding to the strongest components seen in C I and used C I Doppler parameters as priors. The synthetic HD profiles in the UVES spectrum are shown in Fig. 9 together with the corresponding X-shooter profile. We only consider the total HD column density as being reasonably trustable, with $\log N_{\mathrm{HD}}=16.64_{-0.18}^{+0.16}$. This corresponds to $\mathrm{HD} / 2 \mathrm{H}_{2}=\left(8.1_{-2.8}^{+3.7}\right) \times 10^{-5}$, which is significantly higher than typical ratios observed in our Galaxy (Snow et al. 2008). Our inferred ratio is also higher than the primordial value estimated from D I/H I in low metallicity high- $z$ DLAs $\left((\mathrm{D} / \mathrm{H})_{\mathrm{p}}=\right.$ $(2.53 \pm 0.04) \times 10^{-5}$; Cooke et al. 2014). While a high abundance of deuterium can possibly be explained by a strong supply of primordial gas (as suggested by Ivanchik et al. 2010), the molecular ratio observed here is more likely explained by chemical fractionation and charge exchange processes (Liszt 2015). Without entering into details of the HD chemistry, we note that the reaction $\mathrm{D}^{+}+\mathrm{H}_{2} \rightarrow \mathrm{HD}+\mathrm{H}^{+}$is fast and can lead to an increase of $\mathrm{HD}$ compared to $\mathrm{H}_{2}$. If we call $f_{\mathrm{HD}}=\mathrm{HD} /(\mathrm{HD}+\mathrm{D} \mathrm{I})$ the fraction of deuterium in molecular form, then we have

$\frac{\mathrm{HD}}{2 \mathrm{H}_{2}}=\left(\frac{D}{H}\right) \frac{f_{\mathrm{HD}}}{f_{\mathrm{H} 2}}$

Assuming an intrinsic primordial value ${ }^{1}$, the high $\mathrm{HD} / \mathrm{H}_{2}$ ratio can be explained for $f_{\mathrm{HD}} / f_{\mathrm{H} 2} \simeq 3.2$, which naturally requires that the cloud cannot be fully molecular. This is indeed what we conclude from modelling the physical conditions in the cloud (Sect. 6). We however caution that a high-resolution spectrum with high $\mathrm{S} / \mathrm{N}$ ratio is necessary to better take into account blends with the Ly- $\alpha$ forest and confirm our measurement.

We do not take into account astration of the order of 0.1 dex due to
the high metallicity and redshift of our system (see Dvorkin et al. 2016).
Table 5. Neutral chlorine fitting parameters.

\begin{tabular}{ccccc}
\hline \hline zabs & $\begin{array}{c}b \\
\left(\mathrm{~km} \mathrm{~s}^{-1}\right)\end{array}$ & $\log N(\mathrm{Cl} \mathrm{I})$ & $\begin{array}{c}b \\
\left(\mathrm{~km} \mathrm{~s}^{-1}\right)\end{array}$ & $\log N(\mathrm{Cl} \mathrm{I})$ \\
\hline 2.52500 & $6.5 \pm 1.3$ & $13.00 \pm 0.06$ & $6.6 \pm 1.2$ & $13.00 \pm 0.06$ \\
2.52536 & $4.8 \pm 1.5$ & $13.03 \pm 0.10$ & $4.9 \pm 1.1$ & $13.04 \pm 0.06$ \\
2.52546 & $0.8 \pm 0.6$ & $14.55 \pm 1.92$ & $0.7^{a}$ & $14.63 \pm 0.29$ \\
Total & & $14.58 \pm 1.81$ & & $14.65 \pm 0.27$ \\
\hline
\end{tabular}

Notes. ${ }^{(a)}$ Fixed value (see text).

\subsection{Neutral chlorine}

Chlorine is known to be tightly linked with $\mathrm{H}_{2}$ thanks to rapid chemical reactions (e.g. Jura 1974a). In our Galaxy, observations of clouds with $\log N\left(\mathrm{H}_{2}\right)>19$ using the Copernicus satellite have revealed a clear correlation between the column density of both species (Moomey et al. 2012). Recently, Balashev et al. (2015) have used a sample of known $\mathrm{H}_{2}$-bearing DLAs to show that this relation stands at high redshifts and down to ten times lower column densities. Here, only one absorption line of neutral chlorine, $\mathrm{Cl} \mathrm{I} \lambda 1347$ is covered and not blended in our spectrum. Three components, that match those seen in the neutral carbon profile, are detected and used to constrain the column densities, while those associated to the weakest $\mathrm{C}$ I components are below our detection limit. This again indicates that $\mathrm{H}_{2}$ should actually be present in more than one component, although too close to be distinguished within the damped profile of the strong, cold component. Unfortunately, the column density of neutral chlorine in that component is poorly constrained due to this line being in the intermediate regime with a strong dependence on the Doppler parameter. Therefore, the Voigt profile fit, with all parameters kept free, lead to a very high uncertainty in the column density. However, we can make the reasonable assumption that the Doppler parameter should be close to that of other species for this component. Since chlorine is expected to arise from the $\mathrm{H}_{2}$-bearing gas but with a much higher atomic mass, its thermal broadening should be negligible and its Doppler parameter close to that of other "heavy" species. Assuming $b=0.7 \mathrm{~km} \mathrm{~s}^{-1}$ (see Fig. 16), we obtained a very satisfactory fit with $\log N(\mathrm{Cl}$ I $)=$ $14.6 \pm 0.3$ in the narrow component. We also fitted $\mathrm{Cl}$ I assuming a more relaxed constraint on $b$. Taking $b$ to be in the range $0.6-0.8 \mathrm{~km} \mathrm{~s}^{-1}$, gave $\log N(\mathrm{Cl} \mathrm{I})=14.43-14.89$ with a similar uncertainty of 0.3 dex. This sets the overall uncertainty to about 0.4 dex. The results are shown in Fig. 10 and Table 5, where we also provide the fitting results leaving the Doppler parameter totally free, for completeness.

\subsection{Neutral sulphur}

Because the first ionisation potential of sulphur is $10.36 \mathrm{eV}$, neutral sulphur is only expected to be found in very shielded regions. To our knowledge, only a handful detections of S I have been reported so far in DLAs, all associated to a molecular absorber featuring $\mathrm{CO}$ (Srianand et al. 2008) and/or strong $\mathrm{H}_{2}$ absorptions (Milutinovic et al. 2010; Balashev et al. 2011). Here, we detect S I absorption lines with $\log N(\mathrm{~S} \mathrm{I})=14.85 \pm 0.18$ in our UVES spectrum from five transitions in a single narrow $\left(b=0.50 \pm 0.07 \mathrm{~km} \mathrm{~s}^{-1}\right)$ component, see Fig. 11. This suggests that S I can be used as a tracer for CO (Noterdaeme et al. 2010), just like the presence of $\mathrm{C} \mathrm{I}$ implies that of $\mathrm{H}_{2}$. However, because $\mathrm{S}$ I lines have similar strengths and are located in the same spectral region as CO lines, this is of little practical use to identify 


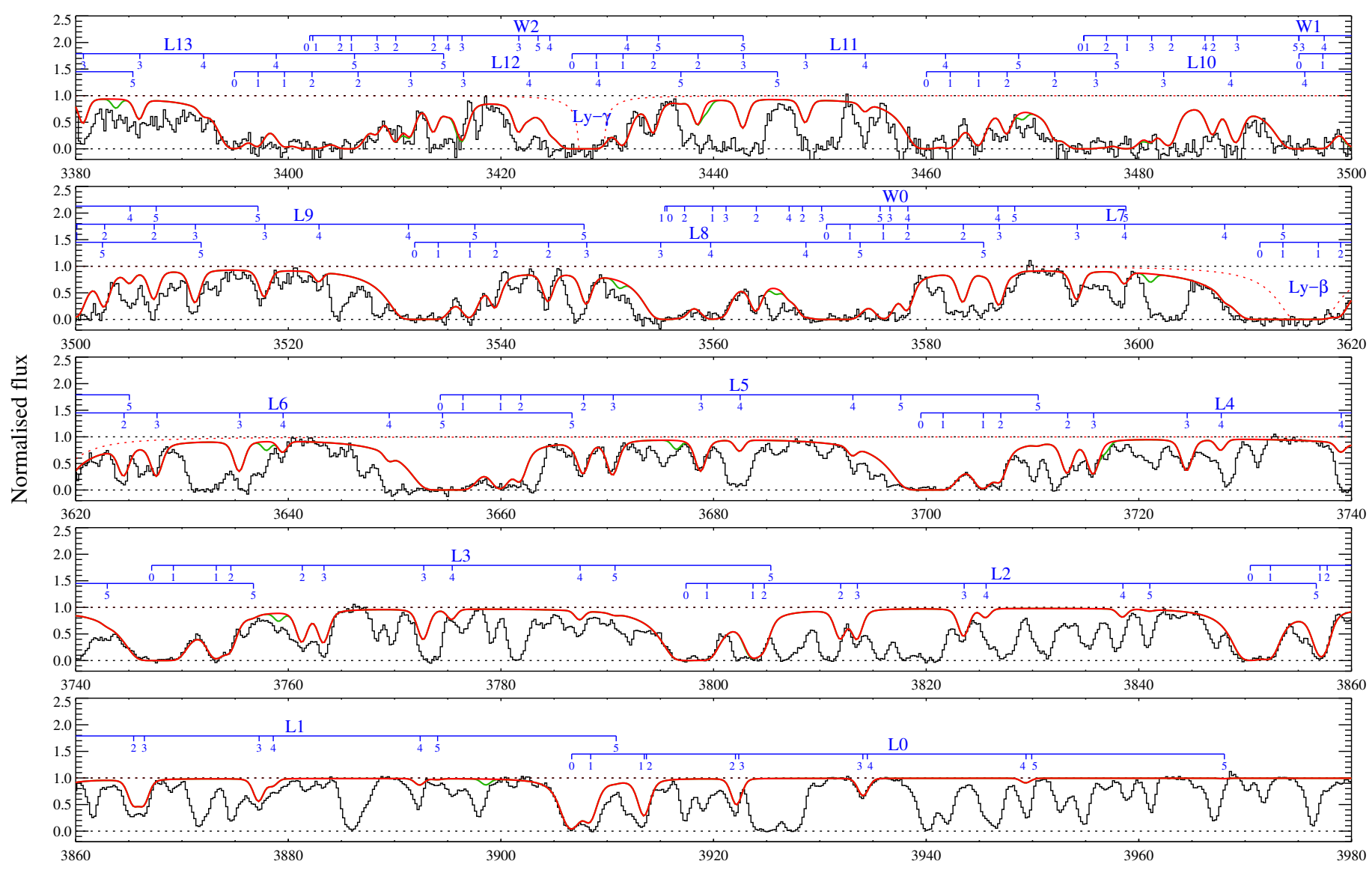

Observed wavelength $(\AA)$

Fig. 8. Portion of X-shooter UVB spectrum (black) around the $\mathrm{H}_{2}$ lines, with the best-fit synthetic spectrum for $\mathrm{H}_{2}$ absorption (with $b=1 \mathrm{~km} \mathrm{~s}{ }^{-1}$ ) in red. Horizontal blue segments connect rotational levels (short tick marks) from a given Lyman (L) or Werner (W) band, as labelled above. $\mathrm{H}$ I Ly $-\beta$ and Ly- $\gamma$ from the DLA are indicated as red dashed profiles. The green profile corresponds to HD lines.

CO systems. Still, S I can be helpful in determining the velocity structure of multi-component $\mathrm{CO}$ absorption systems (e.g. Srianand et al. 2008; Noterdaeme et al. 2009a).

\subsection{Neutral magnesium and neutral sodium}

Neutral magnesium $(\mathrm{MgI})$ is detected in five transitions in our UVES spectrum (see Fig. 12). We clearly detect two components and possibly two additional weak components. The main component, again corresponding to the molecular one at $v=$ $0 \mathrm{~km} \mathrm{~s}^{-1}$, contains more than $80 \%$ of the total column density with $\log N(\mathrm{Mg} \mathrm{I})_{\mathrm{c}}=14.1 \pm 0.1$. Interestingly, the hidden saturation of this component with very small Doppler parameter $(b=$ $\left.0.86 \pm 0.1 \mathrm{~km} \mathrm{~s}^{-1}\right)$ is directly evidenced by its relative strength compared to the second strongest component: both these components have similar observed optical depth for $\operatorname{Mg} I \lambda 2026$, but the former is also seen in transitions with much smaller oscillator strengths.

We also detect the NaI $\lambda \lambda 5891,5897$ doublet in the NIR $\mathrm{X}$-shooter spectrum. The non-Gaussian profile indicates that several components are present, although blended at the achieved spectral resolution (around $R \sim 8500$ ). We therefore used the velocity decomposition of $\mathrm{MgI}$ obtained at high spectral resolution, that is, the redshifts and Doppler parameters of $\mathrm{Na}$ I were fixed to the value previously determined for $\mathrm{Mg} I$ and only the column density was allowed to vary. This assumption led to a good fit of the observed $\mathrm{NaI}$ absorption features
(Fig. 13). We obtained a column density in the main component of $\log (\mathrm{Na} \mathrm{I})_{\mathrm{c}}=15.0 \pm 0.3$ with a large fitting uncertainty due to the line being much narrower than the resolution element. We note that the value is very dependent on the exact normalisation and that the first ionisation potential of sodium $(5.14 \mathrm{eV})$ is even lower than that of magnesium $(7.65 \mathrm{eV})$, meaning that $\mathrm{Na}$ I may arise from deeper regions in the cloud. This means that $\mathrm{Na}$ I column densities should be considered with great caution until a high resolution infra-red spectrum is obtained. Using the empirical correlation observed in the Milky Way between Na I equivalent width and $E(B-V)$ from Poznanski et al. (2012), we expect $A_{V} \sim 0.3$ for their implicitly assumed $R_{V}=3.1$. This is consistent with the value obtained in Sect. 5 .

\subsection{Carbon monoxide}

We detect $\mathrm{CO}$ absorption lines from ten bands, belonging to two systems: the $\mathrm{A}^{1} \Pi\left(v^{\prime}\right)-\mathrm{X}^{1} \Sigma^{+}(0)$ for $v^{\prime}=0$ to 8 and the $\mathrm{d}^{3} \Delta(5)-\mathrm{X}^{1} \Sigma^{+}(0)$ inter-band system, see Fig. 14 . We also tentatively detect the $\mathrm{e}^{3} \Sigma^{-}-\mathrm{X}^{1} \Sigma^{+}$system, although the lines remain too weak to be significant (Fig. 15). Rotational levels are unambiguously detected from $J=0$ to $J=3$. The $J=4$ lines are at the noise level for most bands, but included in the fit. We used the updated molecular data summarised in Daprà et al. (2016). Accurate wavelengths were obtained through calibration by laser and VUV synchrotron studies (Salumbides et al. 2012; Niu et al. 2013, 2015), while oscillator strengths and damping 


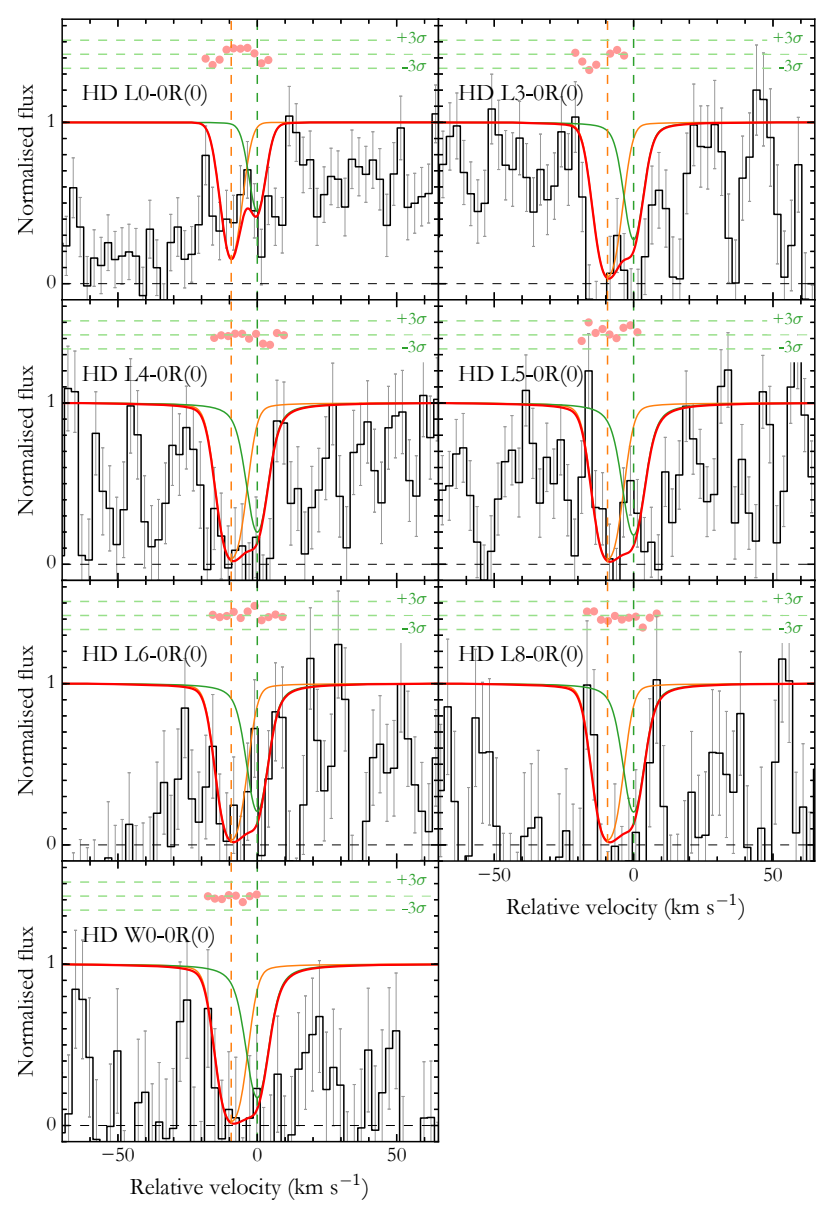

Fig. 9. HD synthetic profile overlaid on the UVES spectrum.

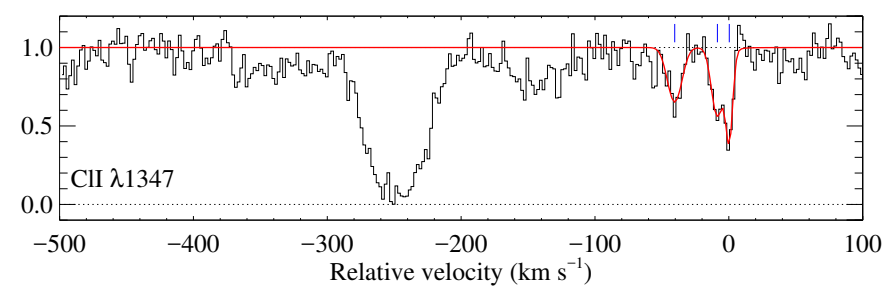

Fig. 10. Fit to the absorption profile of neutral chlorine.

constants were carefully re-evaluated taking into account an updated perturbation analysis. We did not consider the $v^{\prime}=5$ AX system, which was completely blended with the extended Si IV $\lambda 1393$ absorption. Similarly, a large region of the (2-0) absorption band is contaminated by unrelated absorption and ignored during the fitting. Finally, the $(6-0)$ band is also partially blended with smooth absorption from the Ly $\alpha$ forest, but the latter was well modelled using a single component. We therefore included this band and left the intervening Ly $\alpha$ parameters free during the fitting process. The other $\mathrm{CO}$ bands are apparently free from blending. We tied together the Doppler parameter and redshift for the different rotational levels. We were therefore left with seven free parameters for CO: $b, z$ and the column densities for the 5 detected rotational levels. We obtained a satisfactory fit with a global $\chi_{v}^{2}=1.1$, shown as the red profile in Fig. 14, with $z=2.525467, b=0.7 \mathrm{~km} \mathrm{~s}^{-1}$ and obtained a total CO column density of almost $10^{15} \mathrm{~cm}^{-2}$, which is the highest value measured among high- $z$ quasar absorption systems to date. We further tested the robustness of our measurement. The details of this analysis are shown in the appendix.
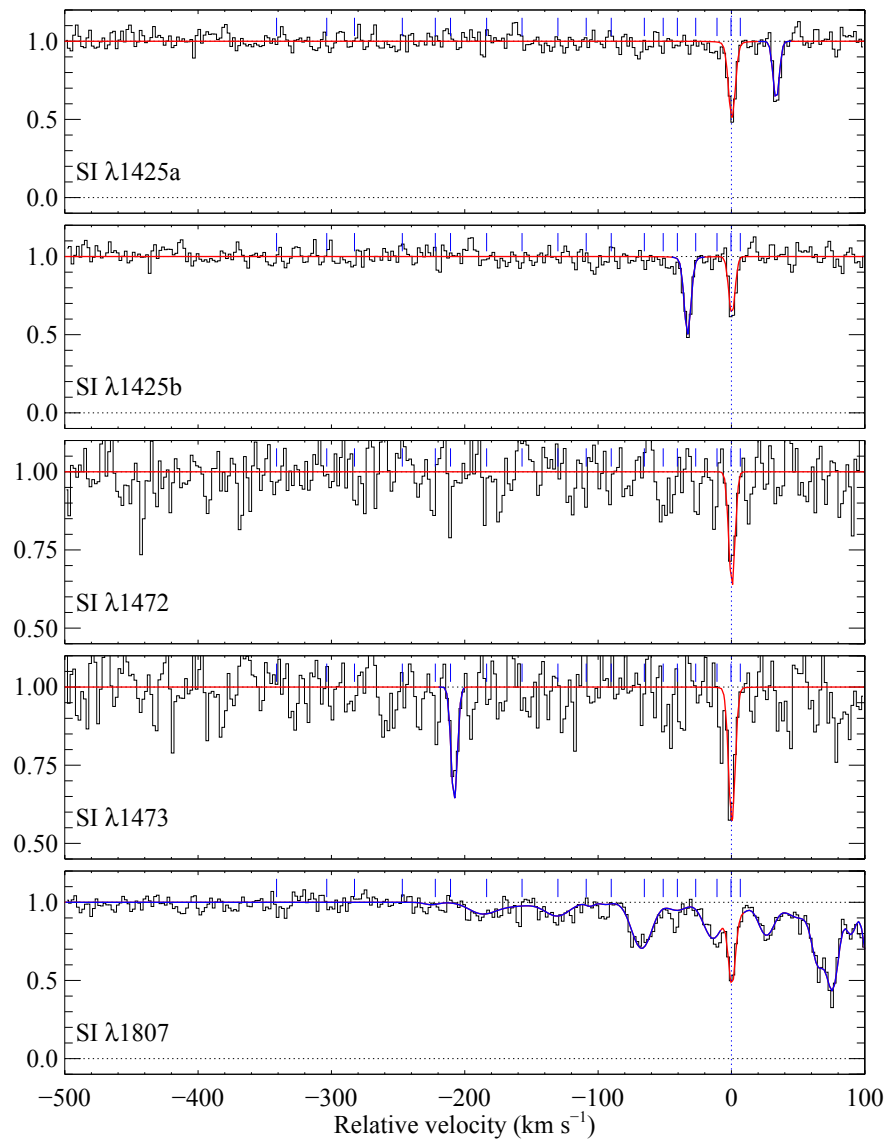

Fig. 11. Neutral sulphur absorption lines (UVES data). As for Fig. 4, the continuous red line correspond to the best fit model, with regions corresponding to other absorption lines marked in blue (for example, most of the profile seen in the S I $\lambda 1807$ actually corresponds to Si II $\lambda 1808$, except around $v=0 \mathrm{~km} \mathrm{~s}^{-1}$ ). The short tick marks show the position of the singly ionised metal lines. We note that $\mathrm{S} I$ is detected only in the component that has $\mathrm{CO}$.

From the non-detection of ${ }^{13} \mathrm{CO}$ lines, we also constrain the isotopic ratio ${ }^{12} \mathrm{CO} /{ }^{13} \mathrm{CO}$ to be higher than 40 , assuming the same Doppler parameter, redshift and excitation temperature for both molecules. This is comparable with values in the solar neighbourhood $\left({ }^{12} \mathrm{CO} /{ }^{13} \mathrm{CO} \sim 70\right.$; Sheffer et al. 2007), meaning that a measurement of the $\mathrm{CO}$ isotopic ratio at high- $z$ should be possible in the near future. This is particularly interesting since the isotopic ratio seems to be anticorrelated with $N(\mathrm{CO})$ in the Galaxy, indicating ${ }^{13} \mathrm{CO}$ enhancement through chemical fractionation in the denser and colder regions (e.g. Sonnentrucker et al. 2007).

\section{Metallicity and dust depletion}

\subsection{Metallicity in the atomic and molecular gas}

In this section, we briefly discuss the metallicity in the different phases. We denote the abundance of a species $\mathrm{M}$ relative to hydrogen as

$[\mathrm{M} / \mathrm{H}] \equiv \log (N(\mathrm{M}) / N(\mathrm{H}))-\log (N(\mathrm{M}) / N(\mathrm{H}))_{\odot}$,

where solar abundances are taken from the photospheric values of Asplund et al. (2009). H corresponds to the total hydrogen, that is, including both neutral (HI) and molecular $\left(\mathrm{H}_{2}\right)$ forms. From our absorption line analysis, we infer 


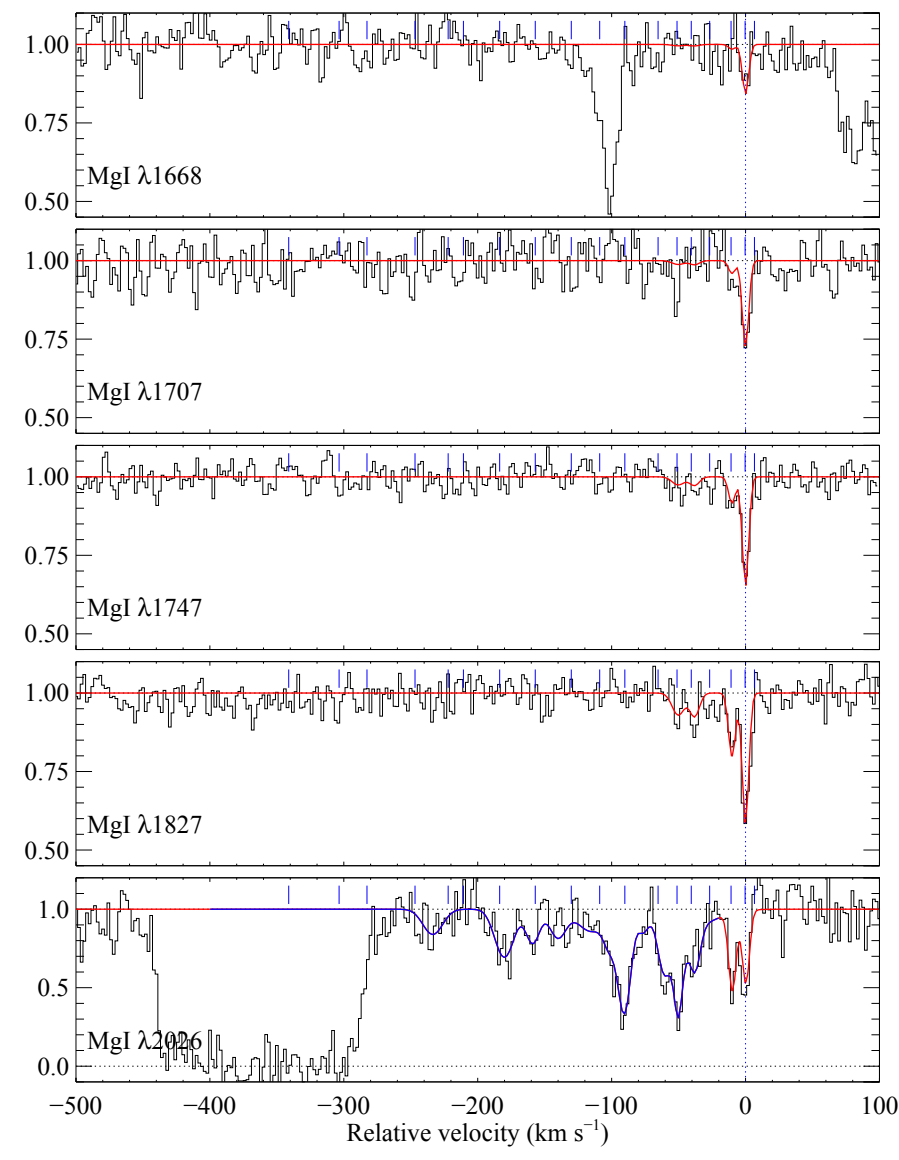

Fig. 12. Fit to the neutral magnesium absorption lines (UVES data). The blue region in the bottom panel corresponds to $\mathrm{Zn}$ II $\lambda 2026$.

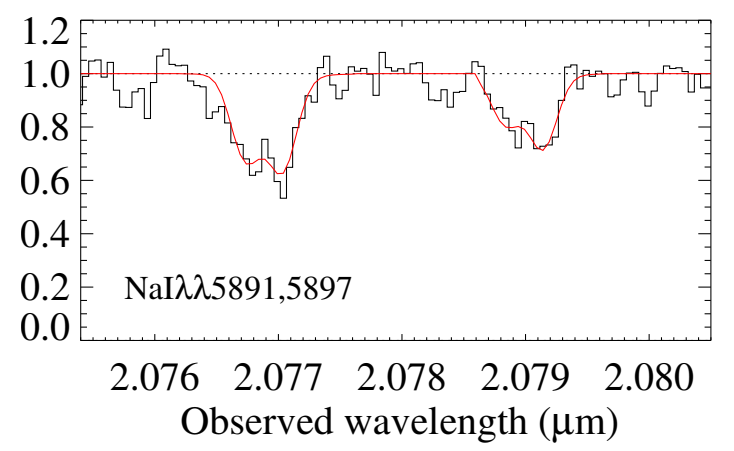

Fig. 13. Portion of the X-shooter NIR spectrum around the Na I doublet. Na I $\lambda 5891$ and NaI $\lambda 5897$ are redshifted at 2.077 and $2.079 \mu \mathrm{m}$, respectively. The red curve corresponds to the Voigt-profile fit using fixed velocity decomposition from $\mathrm{Mg}$ I.

$\log \left(N(\mathrm{HI})+2 N\left(\mathrm{H}_{2}\right)\right)=21.07$. Using the undepleted zinc, we derive an overall super solar metallicity, $[\mathrm{Zn} / \mathrm{H}]=+0.46 \pm 0.45$, where the large uncertainty is due to that on the narrow component, which contains most of the metals. Using phosphorus, we derive $[\mathrm{P} / \mathrm{H}]>-0.04$. This is a lower limit because only the strongest components are detectable and due to some phosphorus depletion in the ISM (by about 0.2 to $0.5 \mathrm{dex}$, see e.g. Lebouteiller et al. 2005). While this is consistent with super-solar metallicity, it also indicates that the upper range from $\mathrm{Zn}$ II is less likely.

It is remarkable that if we assume a molecular fraction of one in the cold component and zero elsewhere, then we get a lower limit to the metallicity of the "warm" gas to be
$[\mathrm{Zn} / \mathrm{H}]_{\mathrm{w}} \geq-0.2 \pm 0.1$, that is, still consistent with solar. We also note that the bluest components $\left(v<-200 \mathrm{~km} \mathrm{~s}^{-1}\right)$ of zinc could not be constrained because of blending with unrelated lines, but these are expected to account for a marginal fraction of the total metal column anyway. Using Si II we obtain a metallicity of $[\mathrm{Si} / \mathrm{H}]_{\mathrm{w}}>-0.6$. This should be considered as a conservative lower limit since silicon depletion is expected to occur (see indeed Sect. 4.2).

While we cannot measure the H I column density in individual components, we can expect that the metallicity in the cold component is at least as high as in the rest of the profile and obtain a more realistic lower limit. We assume

$[\mathrm{Zn} / \mathrm{H}]_{\mathrm{c}} \geq[\mathrm{Zn} / \mathrm{H}]_{\text {overall }}$,

where the index c stands for "cold", that is, associated to the molecule-bearing gas. The molecular fraction in the cold component, $f_{\mathrm{c}}=2 N\left(\mathrm{H}_{2}\right) /\left(2 N\left(\mathrm{H}_{2}\right)+N(\mathrm{HI})_{\mathrm{c}}\right)$ can then be expressed as

$f_{\mathrm{c}} \gtrsim f \frac{N(\mathrm{Zn} \mathrm{II})_{\text {overall }}}{N(\mathrm{Zn} \mathrm{II})_{\mathrm{c}}} \approx 0.54$.

Conversely, if we assume that the cold component is fully molecular (i.e. $f_{\mathrm{c}}=1$ ), we get an upper-limit to the metallicity in that component, $[\mathrm{Zn} / \mathrm{H}]_{\mathrm{c}}<0.7 \pm 0.5$, while the lower-limit to the metallicity in the warm gas is $[\mathrm{Zn} / \mathrm{H}]_{\mathrm{w}}>-0.2$ (see Sect. 3.2).

Because chlorine is associated to the $\mathrm{H}_{2}$-bearing gas, its abundance can also be used to constrain the metallicity of the latter using the relation from Balashev et al. (2015)

$[\mathrm{Cl} / \mathrm{H}]=\left[\mathrm{Cl} / \mathrm{H}_{2}\right]+\log f$,

where

$\left[\mathrm{Cl} / \mathrm{H}_{2}\right]=\log \left(\frac{N(\mathrm{Cl})}{2 N\left(\mathrm{H}_{2}\right)}\right)-\log \left(\frac{\mathrm{Cl}}{\mathrm{H}}\right)_{\odot}$.

We measured $\left[\mathrm{Cl} / \mathrm{H}_{2}\right] \sim 0.4 \pm 0.3$ using the fit with fixed Doppler parameter for the main component. The lower limit to the molecular fraction $f>0.46$ (see Sect. 3.4) then implies $[\mathrm{Cl} / \mathrm{H}]>$ $0.05 \pm 0.3$ in the cold component. This is a conservative limit on the metallicity since several studies have argued for some depletion of chlorine, by about a factor of two (e.g. Moomey et al. 2012, and references therein). The abundance of chlorine is therefore consistent with the super-solar metallicity derived from zinc and phosphorus, assuming an intrinsic solar ratio. We also note that assuming a uniform metallicity across the different components implies that about $95 \%$ of $\mathrm{H}_{2}$ resides in the main component.

\subsection{Dust abundance from depletion of refractory elements}

Ledoux et al. (2003) have revealed the existence of a relation between the presence of molecular hydrogen and both the overall metallicity (see also Petitjean et al. 2006) and the observed depletion factors in DLAs, that is, the probability to detect $\mathrm{H}_{2}$ is higher when the relative abundances of metals (or dust) are high. Noterdaeme et al. (2008) further show that the column density of $\mathrm{H}_{2}$ is strongly related to that of dust, quantified by the column density of iron missing from the gas phase $\left(N\left(\mathrm{Fe}_{\mathrm{dust}}\right)\right.$, Vladilo et al. 2006). However, the metallicity and depletion factors are only indicative of the average values over the whole absorption path probed by the DLA while the $N\left(\mathrm{Fe}_{\text {dust }}\right)$ corresponds to an integrated value. Indeed, metals probe gas over a wide range of physical conditions, making it generally difficult 

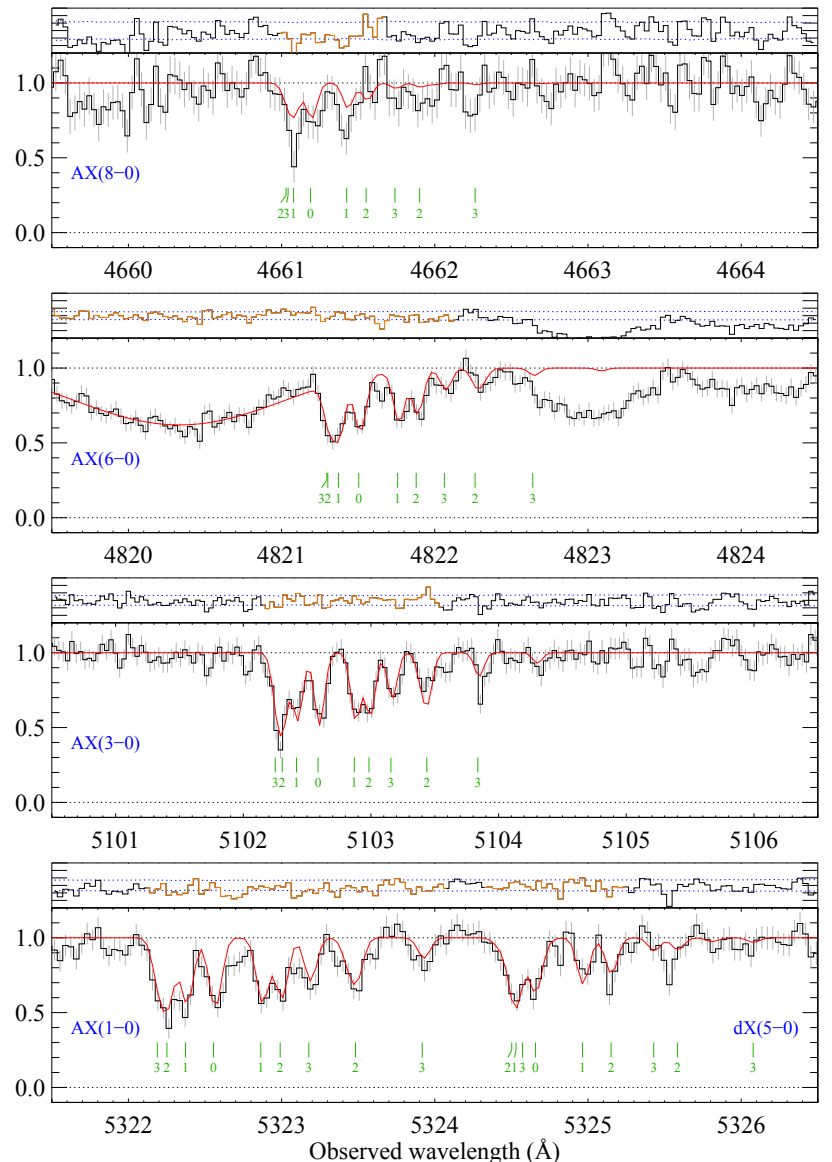
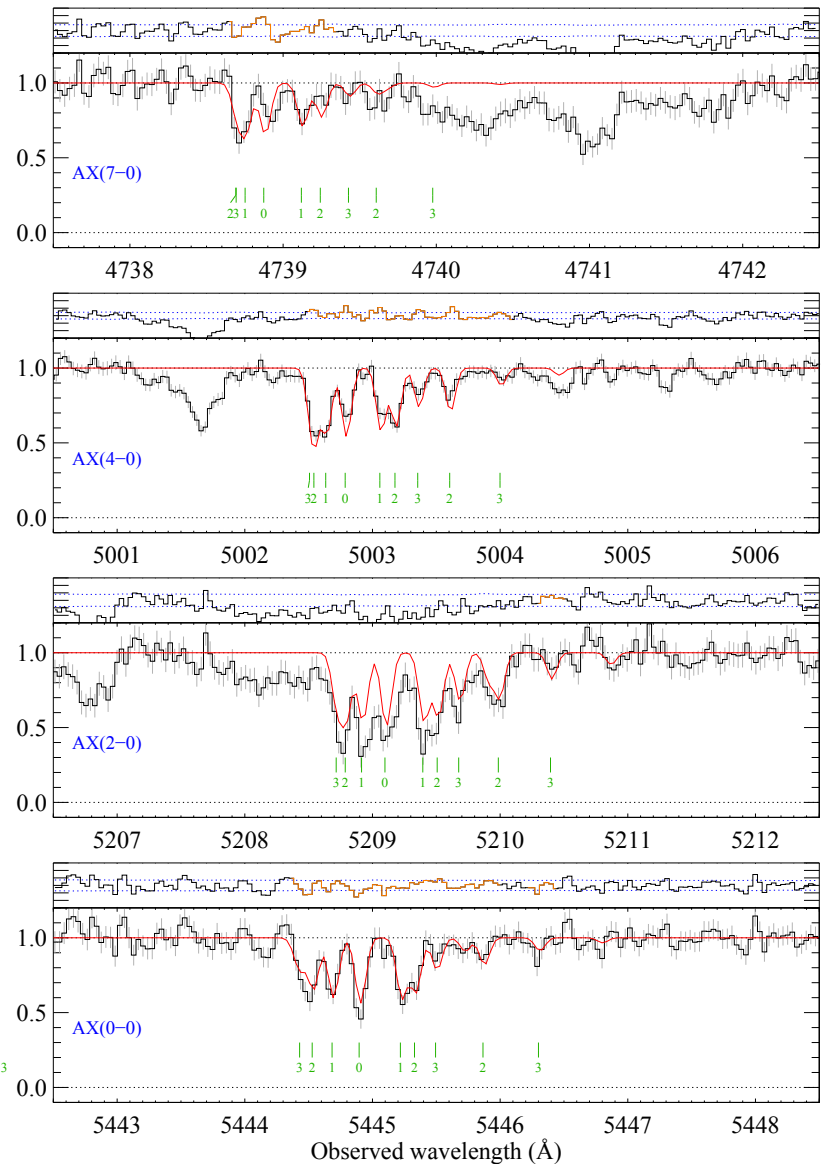

Fig. 14. Voigt-profile fit to the CO absorption bands labelled in blue in each panel (UVES data). Rotational levels from $J=0$ to $J=3$ are indicated as green tick marks. The panel above each region shows the residuals where the blue line indicates the $\pm 1 \sigma$ interval, and the orange regions mark those used to constrain the fit.

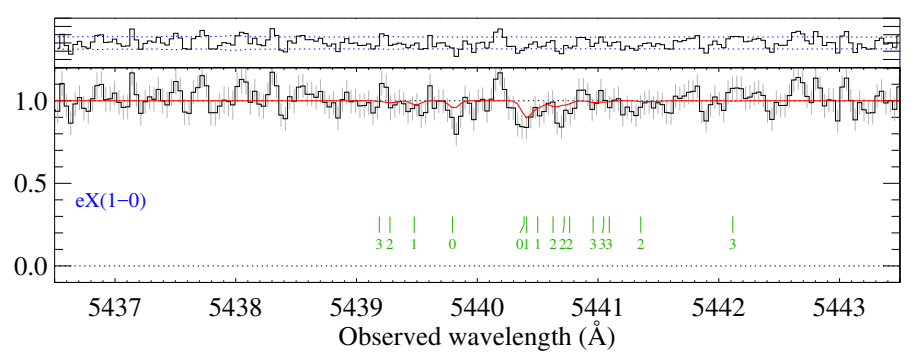

Fig. 15. Tentative detection of $(1-0)$ band of the $\mathrm{e}^{3} \Sigma^{-}-\mathrm{X}^{1} \Sigma^{+} \mathrm{CO}$ system (UVES data). The red profile correspond to the calculated synthetic spectrum, using the parameters obtained from fitting the $\mathrm{AX}$ and $\mathrm{dX}$ bands and molecular data from Eidelsberg \& Rostas (2003).

to associate a given metal component to a molecular one. Still, it has been possible to show that abundance ratios along the velocity profiles tend to show an enhanced depletion factor at the velocity where $\mathrm{H}_{2}$ is detected (Rodríguez et al. 2006).

The system towards $\mathrm{J} 0000+0048$ presents an excellent opportunity to study this further, since the metal profile presents a well defined narrow component corresponding to the molecular gas. Figure 17 presents the observed depletion factors ( $\mathrm{Si}, \mathrm{Ni}$ and Fe relative to $\mathrm{Zn}$ ) component by component. The three patterns follow well each other, indicating that the abundance ratios are mainly dictated by depletion onto dust grains, rather than differential nucleosynthesis. As expected, the cold narrow component presents a high level of dust depletion, indicating that this
Table 6. CO best-fit parameters

\begin{tabular}{ccc}
\hline \hline Rot. level $(J)$ & $\log N(\mathrm{CO}, J)$ & $b\left(\mathrm{~km} \mathrm{~s}^{-1}\right)$ \\
\hline 0 & $14.43 \pm 0.12$ & $0.71 \pm 0.03$ \\
1 & $14.52 \pm 0.08$ & $\prime \prime$ \\
2 & $14.33 \pm 0.06$ & $\prime \prime$ \\
3 & $13.73 \pm 0.05$ & $\prime \prime$ \\
4 & $13.14 \pm 0.13$ & $\prime$ \\
\hline Total & $14.95 \pm 0.05$ & $\prime \prime$ \\
\hline
\end{tabular}

component has a high relative amount of dust. However, a dusty component does not necessarily have a high integrated column density of dust, which will be more naturally related to the column density (and hence detectability) of molecular species. We therefore computed the column density of iron locked into dust grains component by component. The cold, molecule-rich component becomes clearly visible and likely responsible for most of the extinction of the background quasar (see next section). We also notice a secondary peak at $v \approx-40 \mathrm{~km} \mathrm{~s}^{-1}$. Interestingly, this corresponds to the location of a neutral chlorine component, which also likely harbours $\mathrm{H}_{2}$ molecules, though with a lower column density. This suggests that neutral chlorine could be directly used as a "high-resolution" tracer of dust within a DLA. 


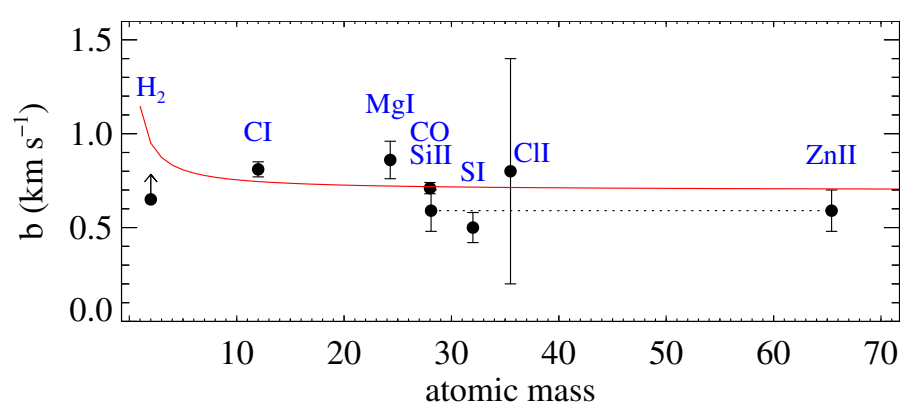

Fig. 16. Doppler parameters and associated $1 \sigma$ uncertainties for different species in the cold component. The red curve represents the expected $b$-value for $T=50 \mathrm{~K}$ and $b_{\text {turb }}=0.7 \mathrm{~km} \mathrm{~s}^{-1}$. We note that the values for Si II and $\mathrm{Zn}$ II were tied together during the fit. The lowerlimit to $b\left(\mathrm{H}_{2}\right)$ corresponds to thermal broadening only.
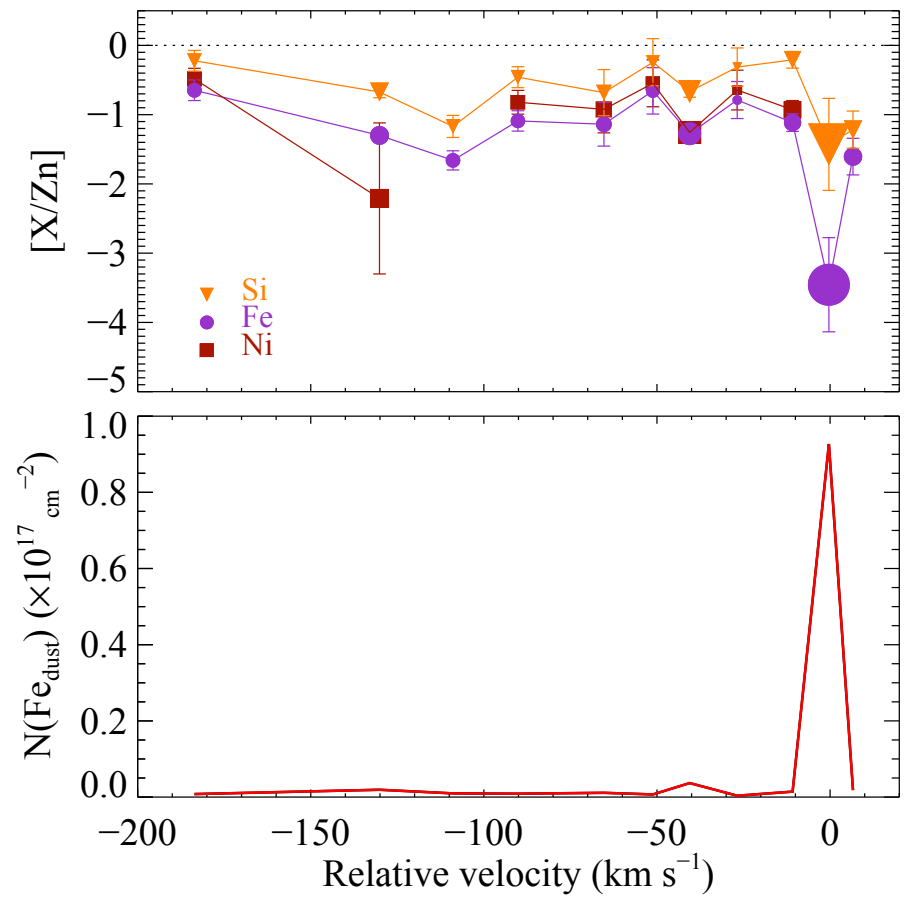

Fig. 17. Top: depletion of silicon, iron and nickel relative to zinc in individual components (left axis). We note that several $[\mathrm{Ni} / \mathrm{Zn}]$ are not available due to the column density of nickel being less than the detection limit. The size of each point is representative of the corresponding $\mathrm{Zn}$ II column density. Bottom: column density of iron locked into dust.

\section{Extinction of the background quasar light}

The spectrum of the quasar J0000+0048 shows clear signs of being reddened, with a clear $2175 \AA$ absorption bump at the redshift of the DLA. This, together with the detection of neutral and molecular species strongly suggests that the dust reddening is caused by the absorber. In the following analysis, we used the quasar template of Selsing et al. (2016) to infer the amount of reddening.

Instead of assuming a fixed extinction curve parametrisation (for example, Small Magellanic Cloud type as typically assumed in the litterature), we were able to constrain the extinction curve towards this quasar in high detail thanks to the long wavelength coverage of X-shooter. For this purpose, we defined continuum regions in the observed spectrum which were not strongly influenced by absorption (both telluric and from the DLA) or broad emission lines. In the near-infrared, we performed a $5 \sigma$ clipping in order to discard outliers introduced by the removal of skylines in the data reduction. We included photometry in the $K$-band from the UKIRT Infrared Deep Sky Survey (UKIDSS) and in band 1 from the Wide-Field Infrared Survey Explorer (WISE). We were not able to include the redder bands from WISE, since the quasar template at this redshift only covers part of band 2 at $4.6 \mu \mathrm{m}$. We observed an offset between the UKIDSS photometry and the SDSS and iWISE photometry. This is most plausibly due to variability of the quasar between the different epochs of observation. In order to correct for this offset, we scaled the spectrum to the $z$-band of the SDSS photometry and subsequently scaled the four UKIDSS bands to match the synthetic photometry calculated from the scaled spectrum.

The template of Selsing et al. was then smoothed with a Gaussian kernel ( $\sigma=7$ pixels) to prevent the noise in the template to falsely fit noise peaks in the real data. In order to take into account the uncertainty in the template, we convolved the errors on the spectrum with the uncertainty estimate from the template.

We then fitted the template to the data using 9 free parameters: 7 parameters to describe the extinction curve shape, a freely varying amount of dust, $A_{V}$, and an arbitrary scale since we do not know the intrinsic brightness prior to reddening.

\subsection{Parametrisation of the extinction law}

We used a slightly modified version of the formalism from Fitzpatrick \& Massa (2007, hereafter, FM2007):

$k(\lambda-V)=E(\lambda-V) / E(B-V)=c_{1}+c_{2} x+c_{3} D$,

where

$D=\frac{x^{2}}{\left(x^{2}-x_{0}^{2}\right)^{2}+x^{2} \gamma^{2}}$,

and $x=\lambda^{-1}$ refers to inverse wavelength in units of $\mu \mathrm{m}^{-1}$ at the absorber rest-frame. This corresponds to a linear component for the whole UV range (defined by $c_{1}$ and $c_{2}$ ) plus a $2175 \AA$ bump, parametrised by $c_{3}, x_{0}$ and $\gamma$. FM2007 also consider a far-UV curvature component parametrised by $c_{4}$ at wavelengths shorter than $c_{5}$ (their Eq. (2)). We did not consider this component here (i.e., we set $c_{4}=0$ ) because we did not have enough data in the FUV. Moreover, the quasar template is more uncertain at very short wavelengths. In addition, preliminary fits to the data indicated that $c_{4}$ was poorly constrained and fully consistent with 0 . We therefore excluded this parameter in the following analysis to simplify the fit without loss of generality.

In the infrared (IR), we used the power-law prescription of FM2007 assuming the correlation between $k_{\mathrm{IR}}$ and $R_{V}$, thus yielding an extinction curve of the form (Eq. (7) of FM2007):

$k(\lambda-V)=\left(-0.83+0.63 \times R_{V}\right) \times x^{1.84}-R_{V}$.

Since this part of the extinction curve is beyond the spectral coverage, we chose to reduce the two original IR parameters by including the correlation between $k_{\mathrm{IR}}$ and $R_{V}$. We used a spline interpolation as in FM2007, in the optical range using one anchor point in the optical to ensure a correct normalisation in the $V$-band. In order to obtain a smooth and continuous transition between the various parts, we included two anchor points in the UV and two in the IR. We used the UV points $U_{1}$ and $U_{2}$ as defined in FM2007 at 2600 and $2700 \AA$, respectively. In the IR, we anchored the spline at 0.75 and $1.0 \mu \mathrm{m}^{-1}$ (similar to the $I_{4}$ and $I_{5}$ points of FM2007). 
Table 7. Derived extinction curve parameters and associated $1 \sigma$ uncertainties.

\begin{tabular}{cc}
\hline \hline Parameter & Best fit value \\
\hline$c_{1}$ & $-2.62 \pm 0.18$ \\
$c_{2}$ & $2.24 \pm 0.13$ \\
$c_{3}$ & $2.70 \pm 0.18$ \\
$x_{0}$ & $4.593 \pm 0.005$ \\
$\gamma$ & $0.85 \pm 0.02$ \\
$R_{V}$ & $4.13 \pm 0.39$ \\
$A_{V}$ & $0.23 \pm 0.01$ \\
$\log (\mathrm{s})$ & $0.043 \pm 0.003$ \\
\hline
\end{tabular}

Finally, we converted the extinction curve from the original formulation in terms of $E(\lambda-V)$ to use $A_{\lambda}$ :

$A_{\lambda} / A_{V}=\frac{1}{R_{V}} E(\lambda-V) / E(B-V)+1$.

\subsection{Fitting the extinction}

We fitted the parameters using a Markov chain Monte Carlo approach as implemented in the python package EMCEE (Foreman-Mackey et al. 2013). This way we were able to include priors and parameter boundaries in a straightforward way. The shape parameters for the $2175 \AA$ bump, $x_{0}$ and $\gamma$, were given quite strong priors, since these parameters are generally observed to be very well behaved in many different environments (Fitzpatrick \& Massa 1990; Gordon et al. 2003). As priors on the two parameters, we used the average values from Gordon et al. (2003), $x_{0}=4.57 \pm 0.01$ and $\gamma=0.94 \pm 0.02$.

In order to give the photometry a more appropriate weight compared to the densely sampled spectral data, we calculated an effective number of pixels per filter. We calculated this quantity by integrating the filter transmission curves scaled to a maximum of 1 and interpolated onto a grid with the same sampling as the spectral data. This way, "pixels" with high transmission are weighted more than "pixels" with low transmission. The uncertainty for each filter was then divided by the square of this number.

The chain was initiated with 100 walkers located at initial locations around the best-fit from a quick $\chi^{2}$ minimisation. We then ran the chain for 1200 iterations and discarded the first 600 as burn-in. From the posterior distributions we obtained the best-fit parameters stated in Table 7. We furthermore provide the inferred $A_{\text {bump }}$, which measures the strength of the $2175 \AA$ bump. This quantity is defined in the following way: $A_{\text {bump }}=\pi c_{3} /\left(2 \gamma R_{V}\right) \times A_{V}$.

The best solution is shown in Fig. 18 where the reddened template is plotted on top of the spectral and photometric data. In Fig. 19, we show the inferred extinction curve. For comparison, we also show the average extinction curves towards the Small Magellanic Cloud (SMC) and Large Magellanic Cloud supershell (LMC2) from Gordon et al. (2003) as well as a Milky-Way extinction curve for the $R_{V}$ measured towards the B-type star $\zeta$ Per by Cardelli et al. (1989).

The uncertainty quoted on the $A_{V}$ from the best fit only includes the formal statistical error. This error is not fully representative as it does not take into account the intrinsic variations of the UV slope of the quasar. The slope of the quasar might vary with respect to the used template spectrum, which would lead us to infer a wrong amount of extinction. We have estimated this systematic effect on our best-fit $A_{V}$ by varying the slope of the used quasar template, by multiplying the template with a powerlaw normalised at $5500 \AA$. We note that this approach is only a rough approximation since the quasar shape is poorly described by a single power-law at all wavelengths. However, from about $1200 \AA$ to $1 \mu \mathrm{m}$ in the quasar rest-frame, this is a reasonable approximation. For each variation in the intrinsic slope, we fitted the data again. In this fitting procedure, we kept the extinction curve parameters fixed, since varying the intrinsic slope and $R_{V}$ simultaneously leads to a completely degenerate fit with nonphysical fit parameters.

For a shallower slope (by $+0.2 \mathrm{dex}$ ), we obtained a bestfit $A_{V}$ of $0.12 \mathrm{mag}$. Conversely, for a steeper slope (smaller by $-0.2 \mathrm{dex}$ ), we recovered a larger best-fit value of $A_{V}=0.34 \mathrm{mag}$. This change in slope is consistent with the average spread of intrinsic slopes observed in the literature (Vanden Berk et al. 2001; Krawczyk et al. 2015). Although the different slopes provide acceptable fits to the data, the best fit is obtained with the original quasar template.

As mentioned above, changing the slope of the template will inevitably change the slope of the recovered extinction curve. Although we cannot fit these two quantities together, we can require the fit to reproduce a value of $R_{V}$ consistent with an average Milky Way sight-line $\left(R_{V}=3.1\right)$, which is obtained for a change in slope of +0.04 dex, which in return yields a best-fit $A_{V}$ of $0.17 \mathrm{mag}$.

\section{Modelling of the physical conditions in the cold cloud}

In this section, we aim at understanding the structure of the cold gas by modelling the physical conditions using the version c13 of the spectral simulation code Cloudy (last described in Ferland et al. 2013). This code performs a self-consistent calculation of the thermal, ionisation and chemical balance of both the gas and dust exposed to a radiation source, with a full treatment of $\mathrm{H}_{2}$ introduced by Shaw et al. (2005). The cloud was assumed to be old enough for all physical processes to be in steady state.

\subsection{Geometry and turbulence}

We considered a plane-parallel geometry with radiation illuminating both surfaces of the cloud. Such geometry has been successfully used to reproduce the physical conditions in typical interstellar clouds (e.g. van Dishoeck \& Black 1986). We considered constant density models and stopped the calculation when reaching the observed $\mathrm{H}_{2}$ column density (instead of $N(\mathrm{HI})$ whose measurement encompasses the whole profile). While $\mathrm{H}_{2}$ is also likely present in more than one component, most of it should be found in the main cold component in which $\mathrm{CO}$ is also found. We considered a turbulent broadening of $0.7 \mathrm{~km} \mathrm{~s}^{-1}$, as derived from the Doppler parameter of heavy elements, see Fig. 16. This is mostly important for its effect on the $\mathrm{CO}$ self-shielding with a negligible effect on $\mathrm{H}_{2}$ due to the strong damping wings.

\subsection{Incident radiation field and cosmic rays}

The Haardt-Madau ionising UV background from both galaxies and quasar (see Haardt \& Madau 2012) was included at the absorber's redshift, and so was the CMB radiation. We also considered the presence of a local source of UV radiation by adding a blackbody radiation with a temperature of $T=40000 \mathrm{~K}$, to simulate the presence of hot stars. We parametrised the intensity 


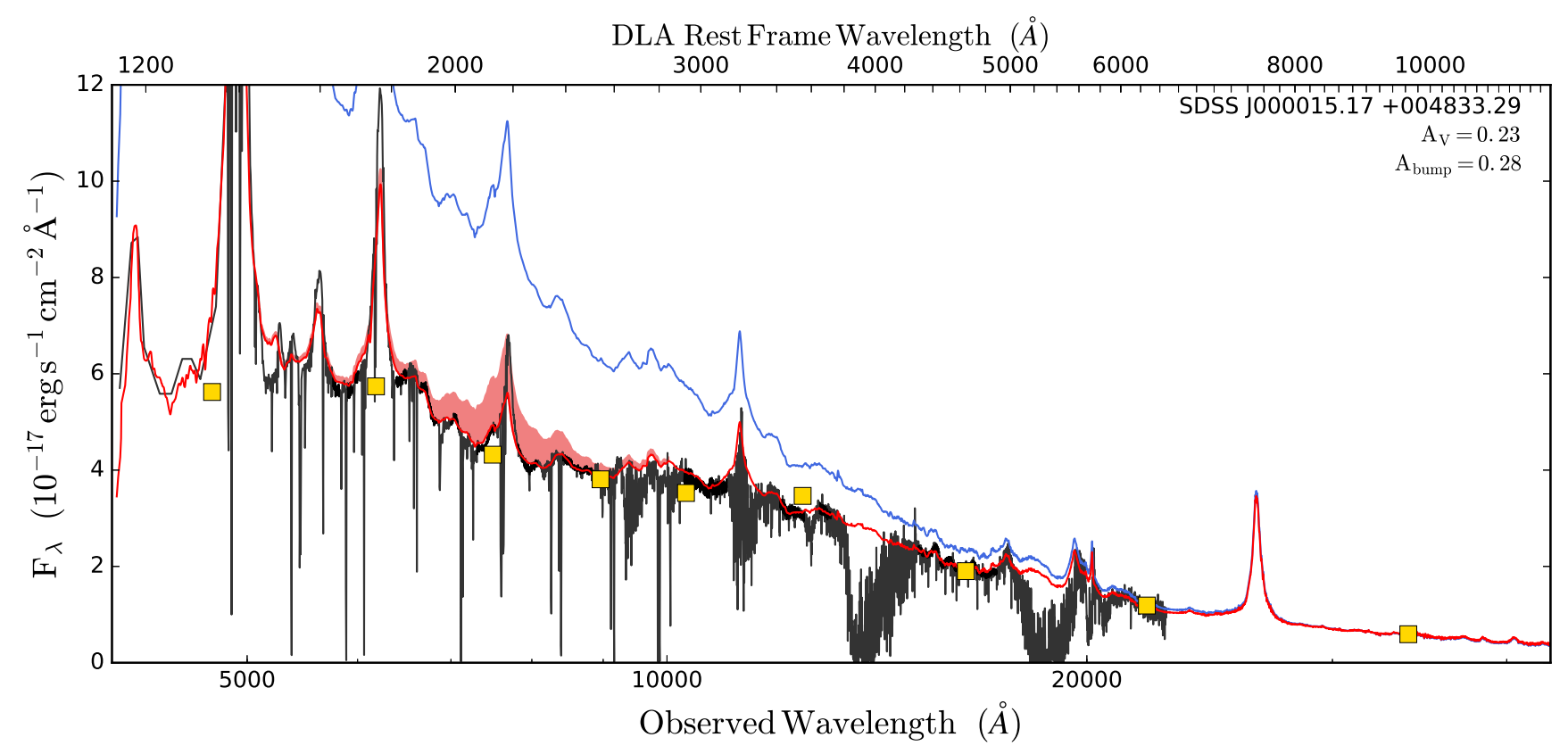

Fig. 18. Combined 1D X-shooter spectrum of J0000+0048 in black. The data has been replaced by the continuum over the Ly $\alpha$ forest. The yellow squares indicate the photometry in $u, g, r, i, z, Y, J, H, K$, and $W l$ bands (left to right). The uncertainty on the photometry is smaller than the extent of the square marker. The blue line shows the unreddened, smoothed template by Selsing et al. (2016), and the red line indicates the same template reddened by the best-fit extinction curve by an amount of $A_{V}=0.23$. The red shaded area marks the strength of the $2175 \AA$ bump. The upper edge of the shaded region corresponds to an extinction curve with no $2175 \AA$ bump.

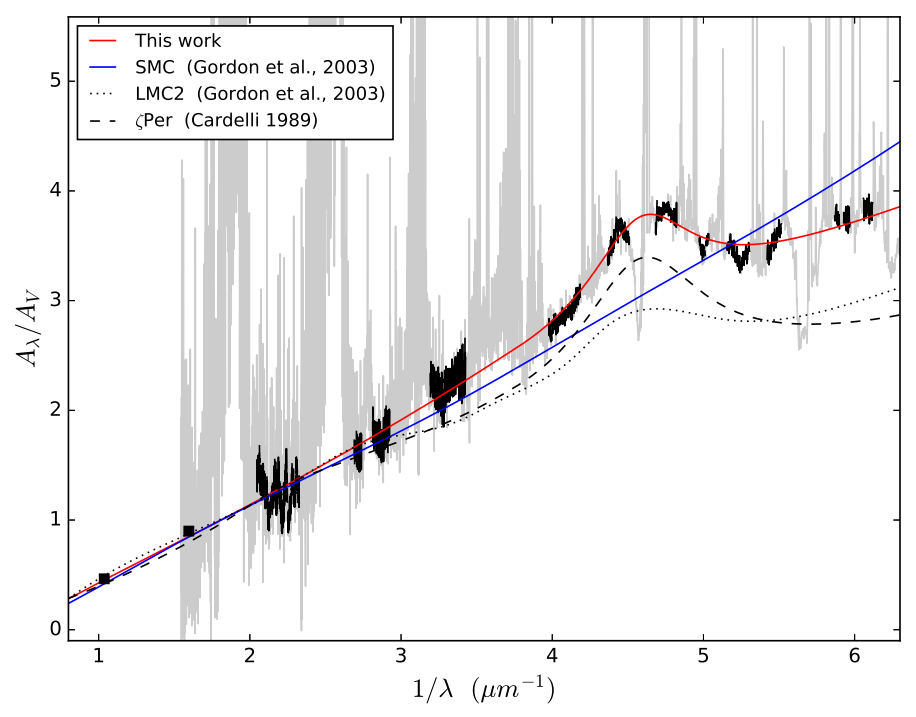

Fig. 19. Extinction curve as function of inverse wavelength at the restframe of the DLA normalised to the $V$-band extinction $A_{V}$. The grey line shows the full $\mathrm{X}$-shooter spectrum divided by the template of Selsing et al. (2016). The black regions indicate the wavelength regions used in the fit, and the two black squares indicated the photometry in the $K$ and $W l$ bands. The red, solid line shows the best fit extinction curve, a clear $2175 \AA$ bump is observed in the data. For comparison, the extinction curves from Gordon et al. (2003) are shown as solid blue (SMC), and grey dotted (LMC2) lines. The dashed line corresponds to a Milky-Way extinction law with the single parameter, $R_{V}$, corresponding to $\zeta$ Per (Cardelli et al. 1989). A full derivation of the extinction curve towards this star is not available.

of this blackbody radiation by $\chi$, the ratio of the assumed incident blackbody radiation to the Habing (1968) field (compared in the range 0.44 to $1 \mathrm{Ryd}$ ). Since we aimed at understanding primarily the conditions in the cold cloud, we took into account the attenuation of the incident radiation after it went through neutral gas, removing photons between 1 to $4 \mathrm{Ryd}$. Cosmic rays were also included as they play a major role inside molecular regions, becoming the main source of ionisation and impacting the ion-molecule chemistry in the cold gas. Indeed, the cosmic ray ionisation rate, $\zeta_{\mathrm{H}}$, is generally deduced from the abundance of chemical ions in our own Galaxy, where it is also found to vary by a large amount between different regions (e.g. Federman et al. 1996). We note however that this remains an active area of research, with more recent studies pointing towards an average Galactic value one order of magnitude higher than previously found (see e.g. Indriolo et al. 2007).

\subsection{Abundances}

We set the metal abundances to 2.5 times solar, as derived from the abundance of undepleted zinc, and assumed intrinsic solar ratio for all species. We applied the observed depletion factor for iron and silicon, which were observed in their dominant ionisation stages. Since we had no measurement of the total abundance of other species, we applied the default depletion values for the cold medium in the Galactic disc as compiled in Table 7.7 of the Hazy1 documentation of Cloudy.

\subsection{Model with standard Galactic grains}

As a first test, we started modelling the cloud using a canonical Milky-Way ISM dust grain mixture, with an abundance scaled to the metallicity, that is, we set the dust grain abundance 2.5 times the Galactic ISM value. Instead of running large grids of parameters, we varied the main input parameters individually and studied their effect on the predicted column densities. These parameters were: total hydrogen volumic density $\left(n_{\mathrm{H}}\right)$, strength of UV field $(\chi)$ and cosmic ray ionisation rate $\zeta_{\mathrm{H}}$. 
The initial density was mostly determined by matching the observed relative population of the $\mathrm{C}$ I fine structure level with the computed ionisation, chemical and thermal balance. We note that the density of different colliders is calculated selfconsistently across the cloud. We found that densities in the range $40-100 \mathrm{~cm}^{-3}$ predict $\mathrm{C}$ I ratios consistent with the ones observed. In turn, the $\mathrm{CO} / \mathrm{C}$ I ratio was strongly under-predicted by a factor of about 30 . This issue has also been raised by Sonnentrucker et al. (2007), who note that most published models of translucent clouds predict less $\mathrm{CO}$ than observed for a given $N\left(\mathrm{H}_{2}\right)$. We found that decreasing $\chi$ did not help (as similarly concluded by Bensch 2006, when modelling the emission from the dark cloud Barnard 5 in the Perseus complex). Shaw et al. (2008) show that the CO column density increases almost linearly with $\zeta_{\mathrm{H}}$; however, increasing $N(\mathrm{CO})$ this way led to a strong overproduction of $\mathrm{C}$ Iin our modelling. We therefore had to re-evaluate our initial assumptions but found that varying other parameters such as the shape of the incident UV field or the geometry of the cloud did not help either.

\subsection{Model with small dust grains}

Interestingly, the abundances of most molecular and neutral species observed at $z_{\mathrm{abs}}=2.5$ towards $\mathrm{J} 0000+0048$ are very similar to those observed in the Perseus cloud along $\zeta$ Per, which has successfully been modelled by Shaw et al. (2008). These authors use a higher number of small grains compared to a standard mixture to approximate the observed $R_{V}$ and $E(B-V)$. The $\zeta$ Per extinction curve is also quite similar to that observed towards J0000+0048, although the latter has a steeper UV slope, probably indicating a smaller average grain size. Small grains seems indeed to be a key ingredient to increase the column density of $\mathrm{CO}$ with respect to that of carbon and molecular hydrogen. In other words, a higher total surface of grains favours $\mathrm{CO}$ production without going too deep into the cloud. Shaw et al. (2016) also highlight the need of increased grain surface area to reproduce the CO column density towards SDSS J1439+1117 (Srianand et al. 2008).

We therefore moved to a second series of models, using a dust grains size distribution containing more small grains. Typically, the distribution for each grain type (graphite and silicates) is parametrised as a single power law with the form $\mathrm{d} n / \mathrm{d} a \propto a^{-q}$, where $n$ is the number of grains with radius in the range $[a$, $a+\mathrm{d} a$ ]. Nozawa \& Fukugita (2013) have shown that a graphitesilicate model can reproduce well the range of extinction curves seen in the Milky-Way and Small Magellanic Cloud with a remarkably constant power law index $q \approx 3.5 \pm 0.2$, with a cutoff at small $\left(a_{\min } \sim 0.05 \mu \mathrm{m}\right)$ and large $\left(a_{\max } \sim 0.2-0.3 \mu \mathrm{m}\right)$ grain radii. These authors note that they could not determine well the small grain cutoff due to lack of data a short wavelengths, but that these have little effect on other parameters anyway. We therefore fixed $q=3.5$ and found that a silicate-graphite mixture with a silicate-to-graphite ratio increased by $40 \%$ compared to canonical ISM mixture, together with $a_{\min }=0.001 \mu \mathrm{m}$ and $a_{\max }=0.15 \mu \mathrm{m}$ for both grain types, reproduced well the extinction curve derived in Sect. 5. In addition, we verified that the abundances of different metals locked into the grains were consistent with the observed metallicity for the assumed depletion pattern to within 0.1 dex, or better.

Using the small-grains model, we were able to reproduce most of the column densities to within 0.4 dex or better for most neutral, ionised, and molecular species, see Table 9, for $\chi=0.5$ and $\zeta_{\mathrm{H}}=2.5 \times 10^{-15} \mathrm{~s}^{-1}$ (Table 8). The column density of Mg I remains however over-predicted by about 0.8 and
Table 8. Cloudy input parameters.

\begin{tabular}{cc}
\hline \hline Parameter & Value \\
\hline$n_{\mathrm{H}}$ & $80 \mathrm{~cm}^{-3}$ \\
$\zeta_{\mathrm{H}}$ & $2.5 \times 10^{-15} \mathrm{~s}^{-1}$ \\
$\chi$ & 0.5 \\
turbulence $^{-1}$ & $0.7 \mathrm{~km} \mathrm{~s}^{-1}$ \\
Metallicity $^{a}$ & $2.5 \mathrm{Z}_{\odot}$ \\
$\log (\mathrm{C} / \mathrm{H})$ & -3.57 \\
$\log (\mathrm{O} / \mathrm{H})$ & -3.13 \\
$\log (\mathrm{Na} / \mathrm{H})$ & -6.06 \\
$\log (\mathrm{Mg} / \mathrm{H})$ & -4.70 \\
$\log (\mathrm{Si} / \mathrm{H})$ & -5.49 \\
$\log (\mathrm{Cl} / \mathrm{H})$ & -6.50 \\
$\log (\mathrm{Fe} / \mathrm{H})$ & -6.50 \\
$\log (\mathrm{Ni} / \mathrm{H})$ & -7.38 \\
\hline
\end{tabular}

Notes. ${ }^{(a)}$ Abundance for all species except those listed below. The gasphase abundance for the listed depleted species correspond to the same intrinsic metallicity after appyling the depletion factors described in the text.

the high rotational levels of $\mathrm{H}_{2}$ are under-predicted. It is likely that the actual depletion of magnesium is much higher than assumed here (see De Cia et al. 2016). Indeed, for the high volumic density and molecular fraction estimated here, we can expect an order of magnitude stronger depletion of magnesium (see Jensen \& Snow 2007), in which case the model perfectly matches the observations. Finally, most of the column density in the high- $J$ levels of $\mathrm{H}_{2}$ could arise mostly from an additional warmer component, which is not modelled here. We also note that the predicted visual extinction $\left(A_{V} \sim 0.7\right)$ is higher than what we derived through SED fitting or seen towards $\zeta$ Per, but within a reasonable factor, given the uncertainties on the measurement and on the intrinsic quasar brightness.

In conclusion, it is remarkable that our model reproduces fairly well the observed quantities with the small number of parameters considered. Further fine-tuning of the parameters could still be performed, and other parameters previously fixed could be varied. For example, a harder UV flux, with black-body temperature of $T \sim 60000 \mathrm{~K}$ increases the $\mathrm{CO}$ column density by about 0.07 dex (hence a better agreement) while changing little the other predicted values. However, such fine-tuning is not the purpose of this paper, given the other uncertainties (in particular on the metallicity) and assumptions involved. We also remind that the true geometry of the cloud and its density profile is likely more complex than assumed. Lastly, we note that the chemistry of $\mathrm{CO}$ is an evolving research field. At the low densities of the ISM, CO can be formed through different paths and sequences of reaction. For example, radiative association between $\mathrm{C}^{+}$and $\mathrm{H}_{2}$ (or with a much lower efficiency with $\mathrm{H}^{0}$ ) leads to $\mathrm{CH}^{+}$, which then reacts with $\mathrm{O}$ to produce $\mathrm{CO}$. Several chemistry networds (such as hydrogen-oxygen) are initiated by cosmic rays, hence their importance in controlling the production of CO. However, as discussed by Goldsmith (2013), other physical phenomena such as shock heating (Elitzur \& Watson 1978) or Alfvén waves (e.g. Federman et al. 1996; Visser et al. 2009) can also raise the temperature of the gas, leading to an increase of $\mathrm{CO}$ abundance. Finally, dissipation of energy from supersonic turbulence can deeply modify the chemistry of the gas (Godard et al. 2009). 
Table 9. Comparison of observed and predicted values in the cold component.

\begin{tabular}{|c|c|c|}
\hline Quantity $^{a}$ & Observed value & Model prediction \\
\hline $\mathrm{HI}$ & $<20.9$ & 20.7 \\
\hline $\mathrm{CO}$ & $14.95 \pm 0.05$ & 14.72 \\
\hline $\mathrm{CI}_{\mathrm{I}}$ & $16.22 \pm 0.07$ & 16.40 \\
\hline $\mathrm{C} \mathrm{I}, J=0$ & $16.10 \pm 0.08$ & 16.19 \\
\hline $\mathrm{CI}, J=1$ & $15.54 \pm 0.14$ & 15.93 \\
\hline $\mathrm{CI}, J=2$ & $14.67 \pm 0.11$ & 15.04 \\
\hline S I & $14.85 \pm 0.18$ & 14.53 \\
\hline $\mathrm{Cl} \mathrm{I}$ & $14.63 \pm 0.29$ & 14.50 \\
\hline $\mathrm{Mg} \mathrm{I}^{b}$ & $14.10 \pm 0.10$ & 14.92 \\
\hline $\mathrm{Na} I$ & $15.00 \pm 0.30$ & 14.51 \\
\hline$T_{01}(\mathrm{~K})$ & $51 \pm 2$ & 56 \\
\hline
\end{tabular}

Notes. ${ }^{(a)}$ Except for $T_{01}$, all quantities correspond to column densities expressed in $\log \left(\mathrm{cm}^{-2}\right) .{ }^{(b)}$ See text for the apparently strong mismatch between model and observations.

\subsection{Characteristics of the cloud}

The top panel of Fig. 20 shows the abundances of neutral atomic hydrogen, molecular hydrogen, neutral chlorine and carbon monoxide relative to the total amount of hydrogen as a function of the depth into the cloud. Only half of the profile is shown, the other half being symmetric with the assumed geometry. We find a total cloud size to be about $4 \mathrm{pc}$ along the line of sight. The line of sight passes quickly through a sharp $\mathrm{H}$ I-to- $\mathrm{H}_{2}$ transition, which is very well traced by neutral chlorine and after which the $\mathrm{H}_{2}$ molecular fraction stays constant at about $50 \%$. This incomplete conversion is due to destruction of $\mathrm{H}_{2}$ in the cloud centre, inside the self-shielding layer (see also Liszt 2015). The abundance of $\mathrm{CO}$ starts to be significant immediately after the $\mathrm{HI}$-to- $\mathrm{H}_{2}$ transition. Indeed the chemical networks leading to the formation of $\mathrm{CO}$ with $\mathrm{H}_{2}$ as starting point are much more efficient than those starting with atomic hydrogen (see Goldsmith 2013). In addition, $\mathrm{H}_{2}$ participates in the shielding of CO, through a few but important overlaps with FUV CO electronic bands (e.g. Glassgold et al. 1985; van Dishoeck \& Black 1988; Bensch 2006; Visser et al. 2009). The abundance of C I does not vary much inside the molecular cloud, and do not directly follow $\mathrm{H}_{2}$ nor $\mathrm{CO}$. This is due to the abundance of $\mathrm{CI}$ being mostly determined by the ionisation and recombination balance. In addition, neutral carbon does neither benefit from $\mathrm{H}_{2}$ shielding nor participate directly in the molecules chemistry (which rather involve $\mathrm{C}^{+}$). The fact that $\mathrm{C}_{\mathrm{I}}$ is considered a tracer for $\mathrm{H}_{2}$ is therefore more indirect: $\mathrm{C}$ I probes the conditions that favour the presence of $\mathrm{H}_{2}$, without locally following the latter within layers of a given molecular cloud.

The temperature (central panel) varies slowly between $\sim 70-80 \mathrm{~K}$ and $48 \mathrm{~K}$ towards the center of the cloud, in agreement with the average temperature measured from the column densities in the first $\mathrm{H}_{2}$ rotational levels. Indeed, we predict $T_{01} \sim 56 \mathrm{~K}$, very close to the measured value. Photoelectric effect on dust grains is the main heating source in the external layers on the cloud while cosmic rays become the main heating source in the cloud center. Heating by $\mathrm{H}_{2}$ photo-dissociation contributes for a small fraction to the total heating and naturally decreases after the $\mathrm{H}$ I-to- $\mathrm{H}_{2}$ transition. Cooling is in turn largely dominated by [C II] $157 \mu \mathrm{m}$ emission. The model predicts $\log N\left(\mathrm{C} \mathrm{II}^{*}\right)=15.64$. Unfortunately, we cannot test this prediction since the measurement is affected by a large uncertainty.
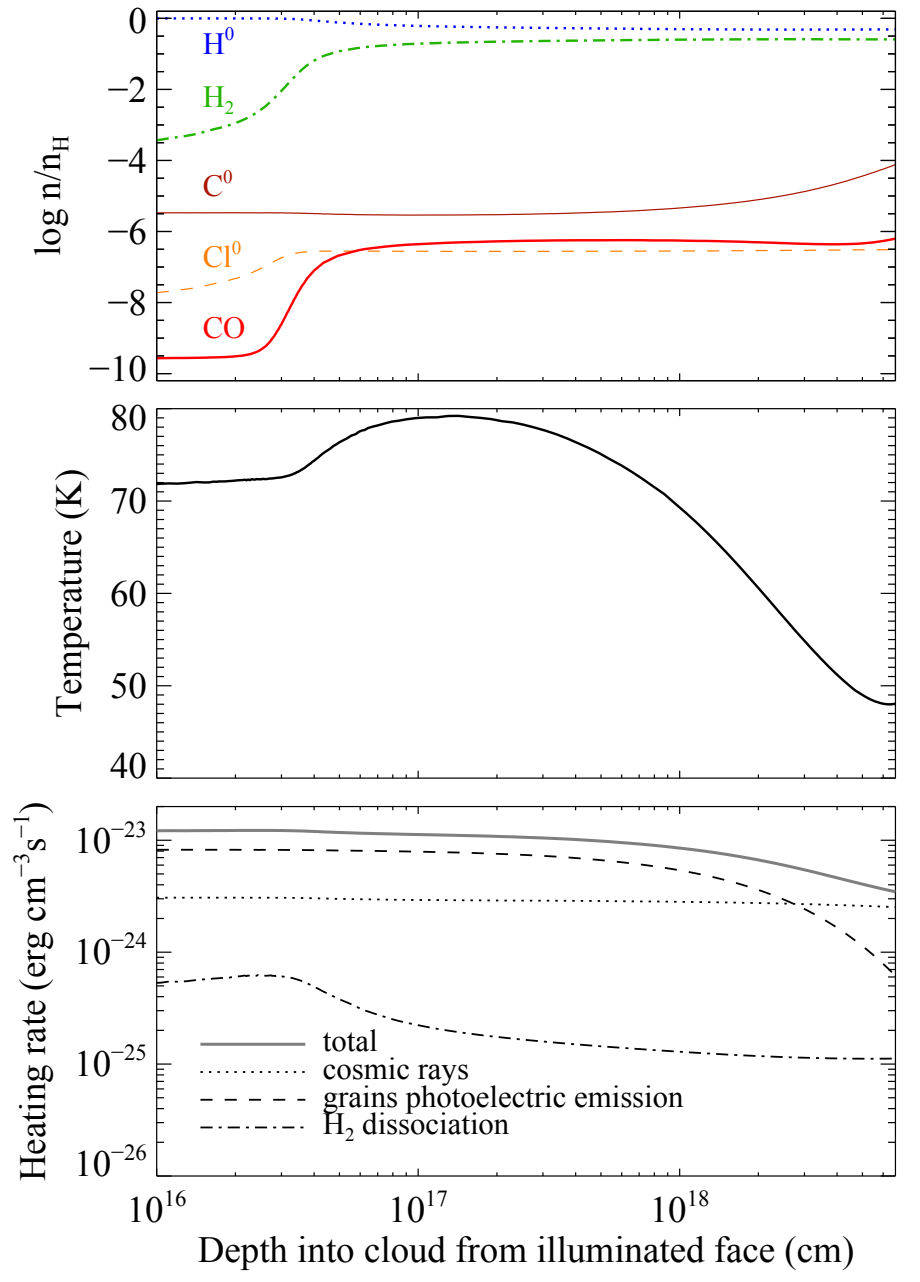

Fig. 20. Top panel: variation of the abundances of neutral atomic hydrogen (blue dotted), molecular hydrogen (green dashed-dotted), neutral chlorine (orange dashed) neutral carbon (brown, dashed-triple dotted) and carbon monoxide (red) as a function of depth into the cloud. We note that for $\mathrm{H}_{2}$, the curve corresponds to half the molecular fraction (since $f=2 n_{\mathrm{H} 2} / n_{\mathrm{H}}$ ). Middle panel: variation of the temperature across the cloud. Bottom panel: heating rates for the main sources. The cooling rate, which is largely dominated by [C II $] 157 \mu \mathrm{m}$ emission, equals the total heating rate since the cloud is assumed in thermal equilibrium.

\section{Temperature of the cosmic microwave background at $z=2.53$}

Srianand et al. (2008) and Noterdaeme et al. (2011) have shown that the excitation of $\mathrm{CO}$ at high redshift is largely dominated by the $\mathrm{CMB}$ radiation, that is, that other excitation processes such as collision are negligible, in which case $T_{\mathrm{ex}}(\mathrm{CO}) \approx T_{\mathrm{CMB}}$. More recently, Sobolev et al. (2015) estimate a correction to apply if we do take into account collisional excitation with hydrogen atoms and $\mathrm{H}_{2}$ molecules. The correction depends almost linearly on the total volumic density and on the kinetic temprature. In addition, since the main collision partner is $\mathrm{H}_{2}$, the correction increases with the molecular fraction. Using $f=0.5, T=50 \mathrm{~K}$ and $n_{\mathrm{H}}=80 \mathrm{~cm}^{-2}$, their Eq. (7) implies an excess temperature of $\Delta T=0.3 \mathrm{~K}$. Applying this correction to the observed $\mathrm{CO}$ excitation temperature towards $\mathrm{J} 0000+0048\left(T_{\mathrm{ex}}(\mathrm{CO})=9.9_{-0.6}^{+0.7} \mathrm{~K}\right.$, see Fig. 21) we obtained $T_{\mathrm{CMB}}(z=2.53)=9.6_{-0.6}^{+0.7} \mathrm{~K}$ in excellent agreement with the expected $\mathrm{CMB}$ temperature in the standard hot Big-Bang theory $\left(T_{\mathrm{CMB}}=T_{0}(1+z)=9.61 \mathrm{~K}\right)$. Using the statistical equilibrium radiative transfer code RADEX 


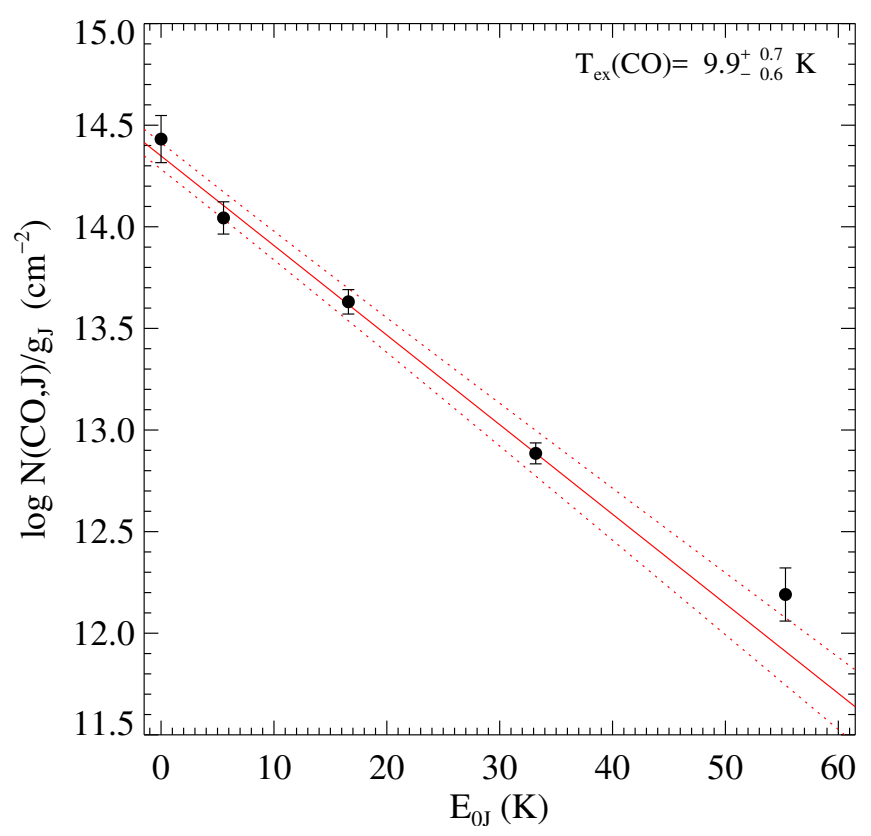

Fig. 21. CO excitation diagram. The solid line gives the best fit to the rotational population using a Boltzmann distribution, with the associated uncertainty shown as dashed line.

(van der Tak et al. 2007), that takes as input the density of various colliders, the kinetic temperature, CO Doppler parameter and column density, we also obtained very similar results, within $0.1 \mathrm{~K}$. We finally remind that since the expected excess temperature is almost linearly dependent on the both kinetic temperature and the density, good constraints on these quantities are highly valuable.

Our Cloudy model predicts $T_{\text {ex }}(\mathrm{CO})=10 \mathrm{~K}$ when the expected CMB radiation field at $z=2.53$ is included, in agreement with the observed temperature. To test further the effect of the $\mathrm{CMB}$ radiation, we ran a model setting the CMB background temperature to the value measured at $z=0$ instead $\left(T_{0}=2.726 \mathrm{~K}\right.$, Fixsen 2009), while keeping all other parameters the same as previously. This model predicts a $\mathrm{CO}$ excitation temperature of $3.8 \mathrm{~K}$, that is derived from the predicted population of the first three rotational levels since higher rotational levels do not follow the Boltzmann distribution anymore. The predicted temperature would then be similar to that seen in diffuse Galactic environments (Burgh et al. 2007) but about $10 \sigma$ away from the measured value towards $\mathrm{J} 0000+0048$. We note that the difference between $\mathrm{CMB}$ temperature and $\mathrm{CO}$ excitation temperature tends to vanish at high redshift, where excitation by CMB becomes strongly dominant over collisional excitation.

Our result suggests ways to improve the constraints on the evolution of the CMB temperature at high redshift. From the distribution of the $\mathrm{CO}$ rotational levels alone, any departure from the Boltzmann distribution will be due to non-CMB (presumably collisionnal) processes. This means that a measurement remains possible using the low rotational levels, as in Noterdaeme et al. (2010). However, a good understanding of the physical conditions in the cloud, and in particular the temperature and density - that can be very effectively constrained by the observations of low- $J \mathrm{H}_{2}$ lines and $\mathrm{CI}$ fine-structure levels - allows one to correct for collisional excitation. The excess temperature for diffuse molecular cloud will typically be of the order of $0.3 \mathrm{~K}$, that is, a few times larger than the expected measurement uncertainty achievable with future high resolution spectographs on extremely large telescopes. This will therefore need to be carefully evaluated.

Cyanogen $(\mathrm{CN})$ is known to be an even better thermometer of the CMB temperature, with negligible contribution from collisions. Very accurate measurements have been performed using absorption spectroscopy towards nearby stars, including observations towards $\zeta$ Per (e.g. Roth \& Meyer 1995; Ritchey et al. 2011). The similarity with our line of sight provides a good hope that $\mathrm{CN}$ lines will also be detectable in systems like $\mathrm{J} 0000+0048$. Indeed, a strong correlation between $N(\mathrm{CO})$ and $N(\mathrm{CN})$ is observed in our Galaxy (e.g. Fig. 18 of Sonnentrucker et al. 2007), with $N(\mathrm{CN})$ of a few times $10^{12} \mathrm{~cm}^{-2}$ at the CO column density of our system. However, obtaining high $\mathrm{S} / \mathrm{N}$ and resolution in the NIR where the CN $\lambda 3875$ lines are redshifted is technically challenging. In the case of J0000+0048, the lines unfortunately fall in a region of low transmission due to water vapour in the atmosphere. This means that the search for similar molecular systems at higher redshift (for example, $z \sim 2.9-3.5$ for the $\mathrm{CN}$ lines to be redshifted in the $H$-band) needs to be continued. The CMB contribution for such systems will also be higher and their constraint on the evolution of CMB temperature stronger.

\section{Direct search for star-formation activity}

Motivated by the similarity between this absorbing cloud and the local ISM, we searched for the emission associated with active star formation. The main indicator of star formation, available to us in the data at hand, is the nebular emission lines from the photo-ionised gas in the vicinity of young stars. The most prominent of these lines in the rest-frame UV and optical are the two principal Balmer lines $(\mathrm{H} \alpha$ and $\mathrm{H} \beta)$ and the two doublets from singly and doubly ionised oxygen ([O II $] \lambda \lambda 3726,3728$ and [O III] $\lambda \lambda 4959,5007)$, which at the redshift of the DLA fall in the NIR spectrum from X-shooter. Unfortunately the $\mathrm{H} \alpha$ line falls in the last order of the NIR spectrum, which is corrupted by strong sky background. We therefore searched for emission from the DLA counterpart using the following lines: $\mathrm{H} \beta$, [O III], and $[\mathrm{O}$ II $]$. However, we did not detect emission from any of the lines.

For each PA, we first subtracted the quasar trace in the 2D spectrum using a similar approach as described in Krogager et al. (2013) in order to search for the faint emission lines. In the following, we have combined the observations taken with a same position angle. We used an elliptical extraction aperture to search for the lines. In the spatial direction, we used a semi-minor axis extent of one full width at half maximum of the spectral trace $(F W H M=5$ pixels $)$. In the spectral direction, we used a semi-major axis of $400 \mathrm{~km} \mathrm{~s}^{-1}$ around the expected location given from the redshift of the DLA. However, we observed no flux in any of the PAs. We derived the detection limit for the [O II] line by modelling 10000 emission line profiles with various line fluxes (drawn randomly in log-space between $1 \times 10^{-18}$ and $1 \times 10^{-16} \mathrm{erg} \mathrm{s}^{-1} \mathrm{~cm}^{-2}$ ), line widths (drawn randomly between 50 and $350 \mathrm{~km} \mathrm{~s}^{-1}$ ), and line strength ratio for the two transitions of the doublet (randomly drawn between 0.35 and 1.5; see Seaton \& Osterbrock 1957). Each model line profile (assumed to be Gaussian) was convolved with the spatial broadening function, determined from the spatial profile of the quasar trace, to create a 2D line model. We subsequently added noise to the 2D line model according to the noise model from the 2D spectrum. For each realisation, we extracted the flux and noise within the elliptical aperture mentioned above. This resulted in a robust limit on the detectable flux within the aperture, given the fact that we do not know the intrinsic line width of the 
P. Noterdaeme et al.: A Perseus-like molecular cloud at $z_{\mathrm{abs}}=2.5$ towards J0000+0048

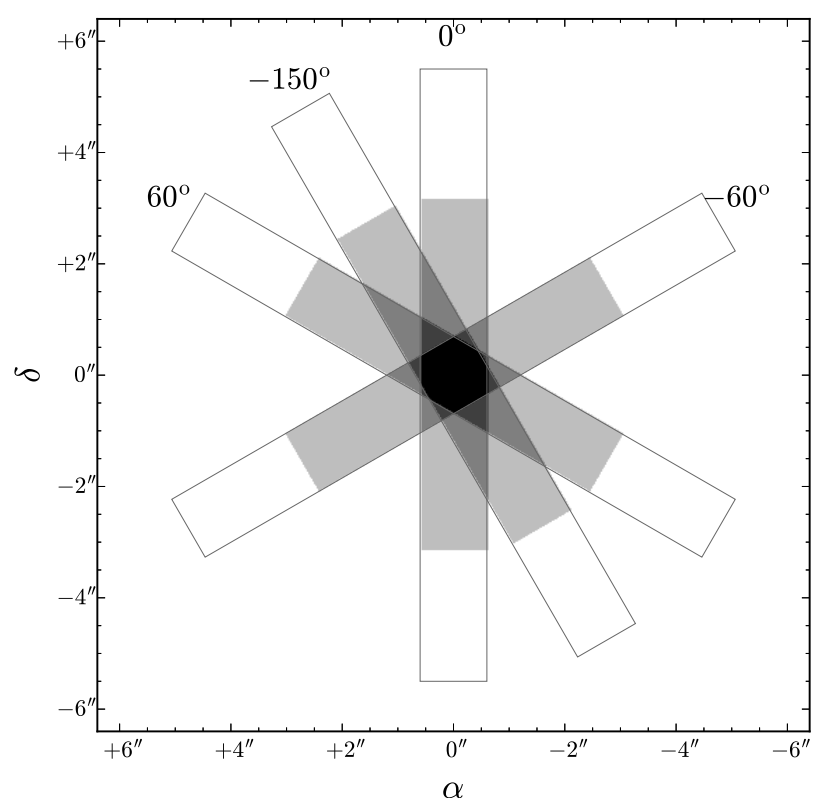

Fig. 22. The positions of the $\mathrm{X}$-shooter slits on the sky relative to the quasar position. The thin grey lines indicate the full X-shooter slit length (11 arcsec). The colour coding indicates effective exposure time in the part of the slit available to our analysis (see text): darker colour corresponds to higher exposure time. For each slit position, the position angle is given at the end of the slit.

[O II] line. We quote all the non-detections for the individual PAs as $3 \sigma$ upper limits in Table 10.

Due to the nodding strategy used for the X-shooter observations, only the central $6.4^{\prime \prime}$ (out of the total $11^{\prime \prime}$ slit length) were suitable for the analysis. However, in the overlap of all the slits, we were able to combine the individual limits to improve the constraint on the line flux. We combined the individual 2D spectra to look at emission but recovered no detection. Using the same elliptical aperture as mentioned above, we measured a $3 \sigma$ limit consistent with the value obtained from the combination of the 4 individual limits. The combined limit measured in the combined stack of all spectra is given in the last row of Table 10. This central region roughly corresponds to a circular aperture with a 1.2 arcsec diameter, which at the redshift of the DLA translates to $5.0 \mathrm{kpc}$. The configuration of slits is shown in the schematic map of exposure time in Fig. 22.

We can use the constraint on the line flux of [O II] $] \lambda 3727$ to put a limit on the star formation rate. Instead of using the relation from Kennicutt (1998), which is calibrated using local galaxies, we used the empirical dust-uncorrected $L[\mathrm{O}$ II $]$-SFR relation from Krühler et al. (2015). This relation has been constructed using high redshift GRB-DLA host galaxies and implicitely accounts for dependence on galactic properties (e.g. Kewley et al. 2004). The inferred star formation rates are summarised along with the flux limits in Table 10.

\section{Summary}

In this work, we presented the detection and detailed analysis of an exceptional molecular absorber at $z_{\mathrm{abs}}=2.53$ towards the quasar $\mathrm{J} 0000+0048$, being one of the very few absorption systems featuring $\mathrm{CO}$ absorption lines known to date (see Noterdaeme et al. 2011, and references therein; Ma et al. 2015). Using both high resolution and multiwavelength spectroscopic observations, we derived the chemical composition, dust
Table 10. Limits on star-formation rate from the non-detection of [O II].

\begin{tabular}{ccc}
\hline \hline $\begin{array}{c}\text { PA } \\
(\mathrm{deg})\end{array}$ & $\begin{array}{c}F([\mathrm{O} \mathrm{II}]) \\
\left(10^{-17} \mathrm{erg} \mathrm{s}^{-1} \mathrm{~cm}^{-2}\right)\end{array}$ & $\begin{array}{c}\mathrm{SFR} \\
\left(M_{\odot} \mathrm{yr}^{-1}\right)\end{array}$ \\
\hline 0 & $<4.0$ & $<22$ \\
-150 & $<3.0$ & $<16$ \\
-60 & $<3.8$ & $<21$ \\
+60 & $<4.3$ & $<24$ \\
combined & $<1.9$ & $<9.9$ \\
\hline
\end{tabular}

depletion and extinction as well as the molecular content of this cloud. Our main findings are the following:

(1) The absorption system is characterised by super-solar metallicity (about $2.5 Z_{\odot}$ ). Although the uncertainty on this value is large (by a factor of about 2-3), it is much higher than the metallicities seen in the overall population of $\mathrm{HI}$-selected DLAs at this redshift (see e.g. Rafelski et al. 2012). The observed depletion of refractory elements is typical of cold gas in the Galactic disc and peaks at the location where molecular gas is found. Higher spectral resolution data would be desirable to constrain better the amount of metals in this very narrow component $\left(b \sim 0.7 \mathrm{~km} \mathrm{~s}^{-1}\right)$.

(2) The DLA has a molecular fraction reaching almost $50 \%$ overall, being the largest value measured till now in a high- $z$ absorption system, for a total neutral hydrogen column density of $\log N(\mathrm{H})=20.8$. This corresponds to a neutral gas surface density of $\Sigma_{\mathrm{HI}} \approx 5 M_{\odot} \mathrm{pc}^{-2}$ and a molecular hydrogen surface density $\Sigma_{\mathrm{H} 2} \approx 4.4 M_{\odot} \mathrm{pc}^{-2}$. These surface densities are very similar to what is seen across the Perseus molecular cloud from high spatial resolution emission observations (Lee et al. 2012), despite the $\mathrm{HI}$ and $\mathrm{H}_{2}$ surface densities being measured directly along a pencil beam line of sight in our study while derived from dust maps in the latter. This also shows that $\mathrm{H}$ I-to- $\mathrm{H}_{2}$ transition in our high- $z$ system is likely following the same physical processes as in our Galaxy.

(3) We further used the different molecular and atomic species to constrain the actual physical conditions in the cold gas with the help of the spectral synthesis code Cloudy. We found that the column densities can be well reproduced by a cloud with density around $n_{\mathrm{H}} \sim 80 \mathrm{~cm}^{-3}$ inmersed into a moderate UV field, similar to the local interstellar radiation field. We showed that a high cosmic ray ionisation rate together with the presence of small dust grains - consistent with the depletion pattern, the steep extinction curve, and presence of a $2175 \AA$ bump - can explain the high CO fractional abundance. The model also predicts a kinetic temperature around $50 \mathrm{~K}$, in perfect agreement with that derived from the excitation of $\mathrm{H}_{2}$. The $\mathrm{CO}$ abundance rises immediately after the $\mathrm{H}$ I-to- $\mathrm{H}_{2}$ transition, thanks to the efficient chemistry paths involving $\mathrm{H}_{2}$, together with pre-shielding of $\mathrm{CO}$. About half of the hydrogen remains in atomic form in the cloud interior, well inside the $\mathrm{H}$ I-to- $\mathrm{H}_{2}$ transition layer, due to $\mathrm{H}_{2}$ destruction by cosmic rays. This can also explain the high $\mathrm{HD} / 2 \mathrm{H}_{2}$ ratio through chemical enhancement of HD compared to $\mathrm{H}_{2}$. We must however keep in mind that, in addition to cosmic rays, several other processes heating the gas can also enhance the production of CO (Goldsmith 2013) and that our model suffers from uncertainties in several imput parameters, such as the metallicity. The detection of more molecular species in this system, together with comparison 
of different codes (e.g. the Meudon PDR code, Le Petit et al. 2006) should break degeneracies between parameters such as dust abundance and cosmic ray rate and lead to a better understanding of the physical processes at play. Our study suggests that the presence of strong $\mathrm{C}$ I lines, detectable at low spectral resolution (as in Ledoux et al. 2015), is a good indicator for high molecular hydrogen column density (in selfshielded regime), but this (or directly detecting damped $\mathrm{H}_{2}$ lines as in Balashev et al. 2014) is not a sufficient condition to get $\mathrm{CO}$ in detectable amounts. Since small grains seem to play a crucial role in the production of $\mathrm{CO}$, selecting systems that, in addition to $\mathrm{C} \mathrm{I}$, also present a steep extinction curve and/or the presence of a $2175 \AA$ bump should significantly increase the probability to detect $\mathrm{CO}$.

(4) While our study shows that the line of sight towards J0000+0048 has chemical and physical characteristics similar to those found in diffuse molecular regions of the Perseus cloud, we have shown that the former absorbing cloud is immersed into a warmer cosmic microwave background radiation. We derived the temperature of the $\mathrm{CMB}$ radiation at the absorber's redshift from the excitation of $\mathrm{CO}$ lines, correcting for a small temperature excess due to collisional excitation. The temperature we obtained is in perfect agreement with the adiabatic cooling expected in the standard hot BigBang theory, $T_{\mathrm{CMB}}(z)=T_{0} \times(1+z) \approx 9.6 \mathrm{~K}$ at $z=2.53$.

Final remarks: the discovery presented here was facilitated by the steadily increasing number of available spectra of faint quasars, together with sensitive instruments on large telescopes. The involved amounts of carbon monoxide remain however far too low to be detectable in emission (even if it were at $z=0$ ), making the cloud being an example of CO-dark molecular gas (Wolfire et al. 2010). Interestingly, such furtive phase may actually contain a large fraction of the total molecular gas in galaxies (e.g. Smith et al. 2014). Efforts should therefore be pursued to detect more absorption systems like the one presented here at high-redshift. These will provide excellent targets for detailled high-resolution studies (including CMB temperature) using the next generation of extremely large telescopes.

Acknowledgements. We warmly thank the anonymous referee for detailed comments and suggestions. We are very grateful to ESO for support during the preparation of OBs, for carrying out the observations in service mode as well as allowing us to accomodate $\mathrm{X}$-shooter observations in an originally UVES progamme (096.A-0924(B)). We thank Robert Carswell for modifying for us his Voigtprofile fitting code VPFIT, providing us an updated version (v10.3) ahead of public release. We also thank Patrick Boissé, Steve Federman and Jacqueline Bergeron for useful discussions during the analysis. We thank Gargi Shaw for help with running Cloudy. P.N. and P.P.J. acknowledge support from the Agence Nationale de la Recherche under grant ANR-12-BS05-0015. P.N., N.G., P.P.J and R.S. acknowledge support from the Indo-French Centre for the Promotion of Advanced Research under project No. 5504-2. J.K. acknowledges support from the European Union's Seventh Framework Programme for research and innovation under the Marie-Curie grant agreement No. 600207 with reference DFF-MOBILEX-5051-00115. S.B. acknowledges support from the Russian Science Foundation under grant 14-12-00955. T.K. acknowledges support through the Sofja Kovalevskaja Award to P. Schady from the Alexander von Humboldt Foundation of Germany. M.T.M. thanks the Australian Research Council for Discovery Project grant DP130100568 which supported this work. This work has made use of the SDSS-III/BOSS database. Funding for SDSS-III has been provided by the Alfred P. Sloan Foundation, the Participating Institutions, the National Science Foundation, and the US Department of Energy Office of Science. The SDSS web site is http://www.sdss.org/. SDSS-III is managed by the Astrophysical Research Consortium for the Participating Institutions of the SDSS-III Collaboration including the University of Arizona, the Brazilian Participation Group, Brookhaven National Laboratory, Carnegie Mellon University, University of Florida, the French Participation Group, the German Participation Group, Harvard University, the Instituto de Astrofisica de Canarias, the Michigan State/Notre Dame/JINA Participation Group, Johns Hopkins University,
Lawrence Berkeley National Laboratory, Max Planck Institute for Astrophysics, Max Planck Institute for Extraterrestrial Physics, New Mexico State University, New York University, Ohio State University, Pennsylvania State University, University of Portsmouth, Princeton University, the Spanish Participation Group, University of Tokyo, University of Utah, Vanderbilt University, University of Virginia, University of Washington, and Yale University.

\section{References}

Albornoz Vásquez, D., Rahmani, H., Noterdaeme, P., et al. 2014, A\&A, 562, A88

Asplund, M., Grevesse, N., Sauval, A. J., \& Scott, P. 2009, ARA\&A, 47, 481

Balashev, S. A., Varshalovich, D. A., \& Ivanchik, A. V. 2009, Astron. Lett., 35 150

Balashev, S. A., Petitjean, P., Ivanchik, A. V., et al. 2011, MNRAS, 418, 357

Balashev, S. A., Klimenko, V. V., Ivanchik, A. V., et al. 2014, MNRAS, 440, 225

Balashev, S. A., Noterdaeme, P., Klimenko, V. V., et al. 2015, A\&A, 575, L8

Bensch, F. 2006, A\&A, 448, 1043

Berry, M., Somerville, R. S., Gawiser, E., et al. 2016, MNRAS, 458, 531

Bialy, S., Sternberg, A., Lee, M.-Y., Le Petit, F., \& Roueff, E. 2015, ApJ, 809, 122

Bigiel, F., Leroy, A., Walter, F., et al. 2008, AJ, 136, 2846

Bird, S., Vogelsberger, M., Haehnelt, M., et al. 2014, MNRAS, 445, 2313

Blitz, L., \& Rosolowsky, E. 2006, ApJ, 650, 933

Bouché, N., Murphy, M. T., Kacprzak, G. G., et al. 2013, Science, 341, 50

Bristow, P., Vernet, J., \& Modigliani, A. 2011, Astron. Nachr., 332, 224

Burgh, E. B., France, K., \& McCandliss, S. R. 2007, ApJ, 658, 446

Cardelli, J. A., Clayton, G. C., \& Mathis, J. S. 1989, ApJ, 345, 245

Carswell, R. F., \& Webb, J. K. 2014, Astrophysics Source Code Library [record ascl: 1408.015]

Carswell, R. F., Becker, G. D., Jorgenson, R. A., Murphy, M. T., \& Wolfe, A. M. 2012, MNRAS, 2703

Catinella, B., Haynes, M. P., Giovanelli, R., Gardner, J. P., \& Connolly, A. J. 2008, ApJ, 685, L13

Cen, R. 2012, ApJ, 748, 121

Chen, Y.-P., Trager, S. C., Peletier, R. F., et al. 2014, A\&A, 565, A117

Ciddor, P. E. 1996, Appl. Opt., 35, 1566

Cooke, R. J., Pettini, M., Jorgenson, R. A., Murphy, M. T., \& Steidel, C. C. 2014 , ApJ, 781, 31

Dalgarno, A., \& Stephens, T. L. 1970, ApJ, 160, L107

Daprà, M., Niu, M. L., Salumbides, E. J., Murphy, M. T., \& Ubachs, W. 2016, ApJ, 826, 192

De Cia, A., Ledoux, C., Mattsson, L., et al. 2016, A\&A, 596, A97

Dekker, H., D’Odorico, S., Kaufer, A., Delabre, B., \& Kotzlowski, H. 2000, in Optical and IR Telescope Instrumentation and Detectors, eds. M. Iye, \& A. F. Moorwood, Proc. SPIE, 4008, 534

Dvorkin, I., Vangioni, E., Silk, J., Petitjean, P., \& Olive, K. A. 2016, MNRAS, 458, L104

Eidelsberg, M., \& Rostas, F. 2003, ApJS, 145, 89

Elitzur, M., \& Watson, W. D. 1978, ApJ, 222, L141

Federman, S. R., Weber, J., Lambert, D. L. 1996, ApJ, 463, 181

Ferland, G. J., Porter, R. L., van Hoof, P. A. M., et al. 2013, Rev. Mex. Astron. Astrofis., 49, 137

Fernández, X., Gim, H. B., van Gorkom, J. H., et al. 2016, ApJ, 824, L1

Field, G. B., Goldsmith, D. W., \& Habing, H. J. 1969, ApJ, 155, L149

Fitzpatrick, E. L., \& Massa, D. 1990, ApJS, 72, 163

Fitzpatrick, E. L., \& Massa, D. 2007, ApJ, 663, 320

Fixsen, D. J. 2009, ApJ, 707, 916

Foreman-Mackey, D., Hogg, D. W., Lang, D., \& Goodman, J. 2013, PASP, 125, 306

Freudling, W., Staveley-Smith, L., Catinella, B., et al. 2011, ApJ, 727, 40

Fukui, Y., \& Kawamura, A. 2010, ARA\&A, 48, 547

Fumagalli, M., O’Meara, J. M., Prochaska, J. X., Rafelski, M., \& Kanekar, N. 2015, MNRAS, 446, 3178

Fynbo, J. P. U., Laursen, P., Ledoux, C., et al. 2010, MNRAS, 408, 2128

Glassgold, A. E., Huggins, P. J., \& Langer, W. D. 1985, ApJ, 290, 615

Godard, B., Falgarone, E., \& Pineau Des Forêts, G. 2009, A\&A, 495, 847

Goldsmith, P. F. 2013, ApJ, 774, 134

Gordon, K. D., Clayton, G. C., Misselt, K. A., Landolt, A. U., \& Wolff, M. J. 2003, ApJ, 594, 279

Haardt, F., \& Madau, P. 2012, ApJ, 746, 125

Habing, H. J. 1968, Bull. Astron. Inst. Netherlands, 19, 421

Hartoog, O. E., Fynbo, J. P. U., Kaper, L., De Cia, A., \& Bagdonaite, J. 2015,

MNRAS, 447, 2738

Horne, K. 1986, PASP, 98, 609

Indriolo, N., Geballe, T. R., Oka, T., \& McCall, B. J. 2007, ApJ, 671, 1736 
Ivanchik, A. V., Petitjean, P., Balashev, S. A., et al. 2010, MNRAS, 404, 1583 Jensen, A. G., \& Snow, T. P. 2007, ApJ, 669, 401

Jorgenson, R. A., Murphy, M. T., Thompson, R., \& Carswell, R. F. 2014, MNRAS, 443, 2783

Jura, M. 1974a, ApJ, 190, L33

Jura, M. 1974b, ApJ, 191, 375

Kanekar, N., Prochaska, J. X., Smette, A., et al. 2014, MNRAS, 438, 2131

Kashikawa, N., Misawa, T., Minowa, Y., et al. 2014, ApJ, 780, 116

Kennicutt, Jr., R. C. 1998, ARA\&A, 36, 189

Kewley, L. J., Geller, M. J., \& Jansen, R. A. 2004, AJ, 127, 2002

Krawczyk, C. M., Richards, G. T., Gallagher, S. C., et al. 2015, AJ, 149, 203

Krogager, J.-K., Fynbo, J. P. U., Møller, P., et al. 2012, MNRAS, 424, L1

Krogager, J.-K., Fynbo, J. P. U., Ledoux, C., et al. 2013, MNRAS, 433, 3091

Krühler, T., Malesani, D., Fynbo, J. P. U., et al. 2015, A\&A, 581, A125

Krumholz, M. R., McKee, C. F., \& Tumlinson, J. 2009, ApJ, 693, 216

Lacour, S., Ziskin, V., Hébrard, G., et al. 2005, ApJ, 627, 251

Lagos, C. D. P., Baugh, C. M., Lacey, C. G., et al. 2011, MNRAS, 418, 1649

Le Petit, F., Nehmé, C., Le Bourlot, J., \& Roueff, E. 2006, ApJS, 164, 506

Lebouteiller, V., Kuassivi, \& Ferlet, R. 2005, A\&A, 443, 509

Ledoux, C., Petitjean, P., \& Srianand, R. 2003, MNRAS, 346, 209

Ledoux, C., Noterdaeme, P., Petitjean, P., \& Srianand, R. 2015, A\&A, 580, A8

Lee, M.-Y., Stanimirović, S., Douglas, K. A., et al. 2012, ApJ, 748, 75

Liszt, H. S. 2015, ApJ, 799, 66

Ma, J., Caucal, P., Noterdaeme, P., et al. 2015, MNRAS, 454, 1751

Milutinovic, N., Ellison, S. L., Prochaska, J. X., \& Tumlinson, J. 2010, MNRAS 408, 2071

Modigliani, A., Goldoni, P., Royer, F., et al. 2010, in SPIE Conf. Ser., 7737

Møller, P., \& Warren, S. J. 1993, A\&A, 270, 43

Møller, P., Fynbo, J. P. U., \& Fall, S. M. 2004, A\&A, 422, L33

Moomey, D., Federman, S. R., \& Sheffer, Y. 2012, ApJ, 744, 174

Murphy, M. T., \& Bernet, M. L. 2016, MNRAS, 455, 1043

Neeleman, M., Prochaska, J. X., \& Wolfe, A. M. 2015, ApJ, 800, 7

Niu, M. L., Salumbides, E. J., Zhao, D., et al. 2013, Mol. Phys., 111, 2163

Niu, M. L., Ramirez, F., Salumbides, E. J., \& Ubachs, W. 2015, J. Chem. Phys., 142,044302

Noterdaeme, P., Ledoux, C., Petitjean, P., et al. 2007, A\&A, 474, 393

Noterdaeme, P., Petitjean, P., Ledoux, C., Srianand, R., \& Ivanchik, A. 2008 A\&A, 491, 397

Noterdaeme, P., Ledoux, C., Srianand, R., Petitjean, P., \& Lopez, S. 2009a, A\&A, 503,765

Noterdaeme, P., Petitjean, P., Ledoux, C., \& Srianand, R. 2009b, A\&A, 505, 1087

Noterdaeme, P., Petitjean, P., Ledoux, C., et al. 2010, A\&A, 523, A80

Noterdaeme, P., Petitjean, P., Srianand, R., Ledoux, C., \& López, S. 2011, A\&A, 526, L7

Noterdaeme, P., López, S., Dumont, V., et al. 2012a, A\&A, 542, L33

Noterdaeme, P., Petitjean, P., Carithers, W. C., et al. 2012b, A\&A, 547, L1

Noterdaeme, P., Petitjean, P., Pâris, I., et al. 2014, A\&A, 566, A24

Noterdaeme, P., Petitjean, P., \& Srianand, R. 2015a, A\&A, 578, L5

Noterdaeme, P., Srianand, R., Rahmani, H., et al. 2015b, A\&A, 577, A24

Nozawa, T., \& Fukugita, M. 2013, ApJ, 770, 27

Petitjean, P., Ledoux, C., Noterdaeme, P., \& Srianand, R. 2006, A\&A, 456, L9

Pettini, M., Smith, L. J., King, D. L., \& Hunstead, R. W. 1997, ApJ, 486, 665

Poznanski, D., Prochaska, J. X., \& Bloom, J. S. 2012, MNRAS, 426, 1465
Prochaska, J. X., \& Wolfe, A. M. 2009, ApJ, 696, 1543

Prochaska, J. X., Tripp, T. M., \& Howk, J. C. 2005, ApJ, 620, L39

Rachford, B. L., Snow, T. P., Tumlinson, J., et al. 2002, ApJ, 577, 221

Rafelski, M., Wolfe, A. M., Prochaska, J. X., Neeleman, M., \& Mendez, A. J. 2012, ApJ, 755, 89

Rahmani, H., Srianand, R., Noterdaeme, P., \& Petitjean, P. 2010, MNRAS, 409, L59

Rao, S. M., Turnshek, D. A., \& Nestor, D. B. 2006, ApJ, 636, 610

Reach, W. T., Koo, B.-C., \& Heiles, C. 1994, ApJ, 429, 672

Ritchey, A. M., Federman, S. R., \& Lambert, D. L. 2011, ApJ, 728, 36

Rodríguez, E., Petitjean, P., Aracil, B., Ledoux, C., \& Srianand, R. 2006, A\&A, 446, 791

Roth, K. C., \& Meyer, D. M. 1995, ApJ, 441, 129

Roy, N., Chengalur, J. N., \& Srianand, R. 2006, MNRAS, 365, L1

Salumbides, E. J., Niu, M. L., Bagdonaite, J., et al. 2012, Phys. Rev. A, 86, 022510

Schlafly, E. F., \& Finkbeiner, D. P. 2011, ApJ, 737, 103

Seaton, M. J., \& Osterbrock, D. E. 1957, ApJ, 125, 66

Selsing, J., Fynbo, J. P. U., Christensen, L., \& Krogager, J.-K. 2016, A\&A, 585, A87

Shaw, G., Ferland, G. J., Abel, N. P., Stancil, P. C., \& van Hoof, P. A. M. 2005, ApJ, 624, 794

Shaw, G., Ferland, G. J., Srianand, R., et al. 2008, ApJ, 675, 405

Shaw, G., Rawlins, K., \& Srianand, R. 2016, MNRAS, 459, 3234

Sheffer, Y., Rogers, M., Federman, S. R., Lambert, D. L., \& Gredel, R. 2007, ApJ, 667, 1002

Smith, R. J., Glover, S. C. O., Clark, P. C., Klessen, R. S., \& Springel, V. 2014, MNRAS, 441, 1628

Snow, T. P., \& McCall, B. J. 2006, ARA\&A, 44, 367

Snow, T. P., Ross, T. L., Destree, J. D., et al. 2008, ApJ, 688, 1124

Sobolev, A. I., Ivanchik, A. V., Varshalovich, D. A., \& Balashev, S. A. 2015, J. Phys. Conf. Ser., 661, 012013

Sonnentrucker, P., Welty, D. E., Thorburn, J. A., \& York, D. G. 2007, ApJS, 168, 58

Srianand, R., Petitjean, P., Ledoux, C., Ferland, G., \& Shaw, G. 2005, MNRAS, 362,549

Srianand, R., Noterdaeme, P., Ledoux, C., \& Petitjean, P. 2008, A\&A, 482, L39

Srianand, R., Gupta, N., Petitjean, P., et al. 2012, MNRAS, 421, 651

Srianand, R., Hussain, T., Noterdaeme, P., et al. 2016, MNRAS, 460, 634

Sternberg, A., Le Petit, F., Roueff, E., \& Le Bourlot, J. 2014, ApJ, 790, 10

Tacconi, L. J., Neri, R., Genzel, R., et al. 2013, ApJ, 768, 74

Tumlinson, J., Shull, J. M., Rachford, B. L., et al. 2002, ApJ, 566, 857

van der Tak, F. F. S., Black, J. H., Schöier, F. L., Jansen, D. J., \& van Dishoeck,

E. F. 2007 , A\&A, 468, 627

van Dishoeck, E. F., \& Black, J. H. 1986, ApJS, 62, 109

van Dishoeck, E. F., \& Black, J. H. 1988, ApJ, 334, 771

Vanden Berk, D. E., Richards, G. T., Bauer, A., et al. 2001, AJ, 122, 549

Visser, R., van Dishoeck, E. F., \& Black, J. H. 2009, A\&A, 503, 323

Vladilo, G., Centurión, M., Levshakov, S. A., et al. 2006, A\&A, 454, 151

Welty, D. E., Xue, R., \& Wong, T. 2012, ApJ, 745, 173

Wolfe, A. M., Howk, J. C., Gawiser, E., Prochaska, J. X., \& Lopez, S. 2004, ApJ, 615,625

Wolfe, A. M., Gawiser, E., \& Prochaska, J. X. 2005, ARA\&A, 43, 861

Wolfire, M. G., Hollenbach, D., \& McKee, C. F. 2010, ApJ, 716, 1191 


\section{Appendix A: Robustness of the $\mathrm{CO}$ measurement}

When estimating best-fit parameters, VPFIT takes as input the normalised spectrum and the resolution provided by the user. This means that continuum placement uncertainties and errors due to the knowledge of the spectral point spread function (SPSF) are not reflected in the error estimates.

We tested the effect of SPSF uncertainties by refitting the data, using a range of spectral resolution from 4.75 to $5.25 \mathrm{~km} \mathrm{~s}^{-1}$. The resulting parameters remain well within their associated fitting uncertainty, with total column density varying by only \pm 0.05 dex, excitation temperature (see Fig. 21) varying by $\Delta T_{\mathrm{ex}} \sim 0.1 \mathrm{~K}$ and Doppler parameter basically unchanged $(<2 \%)$. We also fitted the two red spectra simultaneously, instead of combining them (Fig. A.1). The results are also very close to what we get from the combined spectrum, as can be seen from the blue and red error bars in Fig. A.2, with a slight improvement in the $\chi_{v}^{2}=1.03$ probably thanks to the better evaluated SPSF.
To estimate the effect of continuum placement uncertainties, we independently renormalised each region around $\mathrm{CO}$ absorption bands by randomly modifying the local continuum slope and intercept, following their respective normal error distribution. The distribution of CO column densities, Doppler parameter and excitation temperature obtained for hundred realisations of this "shaky" continuum procedure is shown in Fig. A.2. Clearly, the effect of continuum placement uncertainty is well within the error estimates from fitting the lines but highlights the correlation between $b, N$ and $T_{\text {ex }}$. For example, a smaller Doppler parameter will result in a higher column density and a lower excitation temperature. It can also be seen from this figure that the most deviant points generally also have the highest $\chi_{v}^{2}$-values. 
P. Noterdaeme et al.: A Perseus-like molecular cloud at $z_{\mathrm{abs}}=2.5$ towards J0000+0048

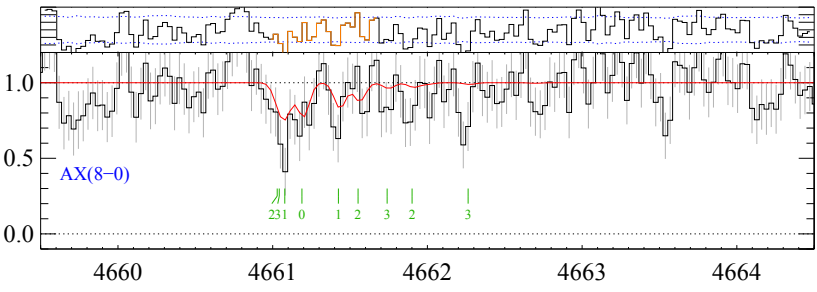

唇

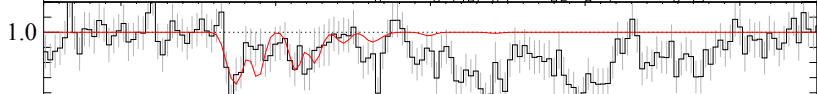
0.5 E

$0.0=$

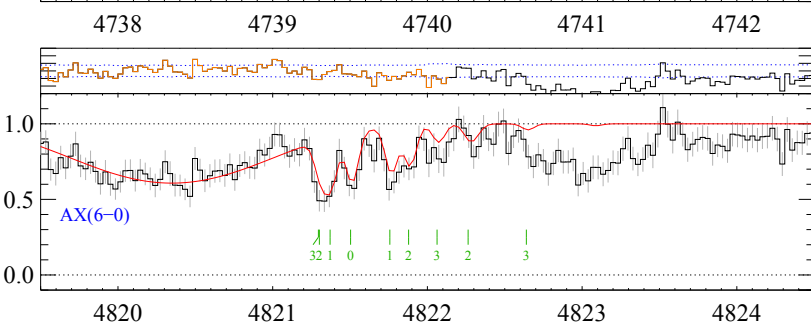

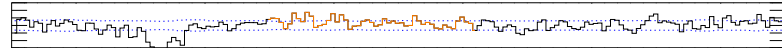

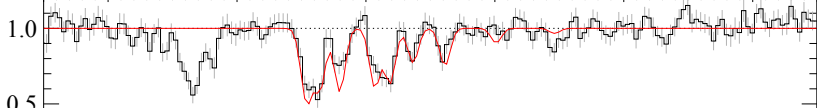
$0.5 E_{\mathrm{AX}(4-0)}$

0.0

$\begin{array}{llllll}5001 & 5002 & 5003 & 5004 & 5005 & 5006\end{array}$

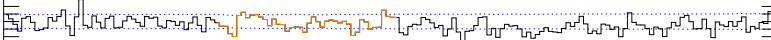
0.0

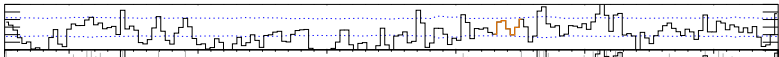
1.0 年 $0.5=-\mathrm{AX}(2-0)$ $0.0=$

$\begin{array}{llllll}5207 & 5208 & 5209 & 5210 & 5211 & 5212\end{array}$

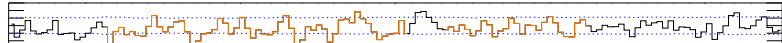
$0.5=2$ Ex(1-0) 0.0

$$
322
$$$$
\begin{array}{lll}
211 ! \\
2130
\end{array}
$$

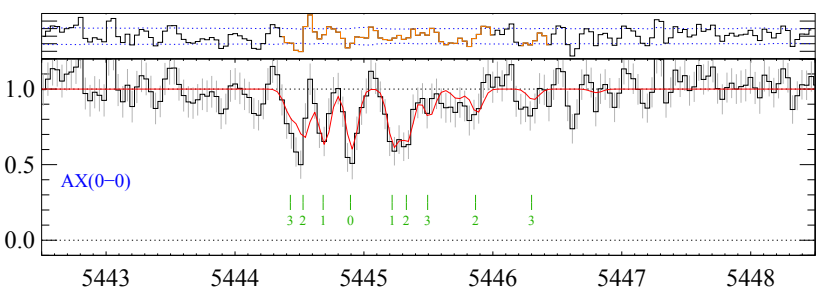

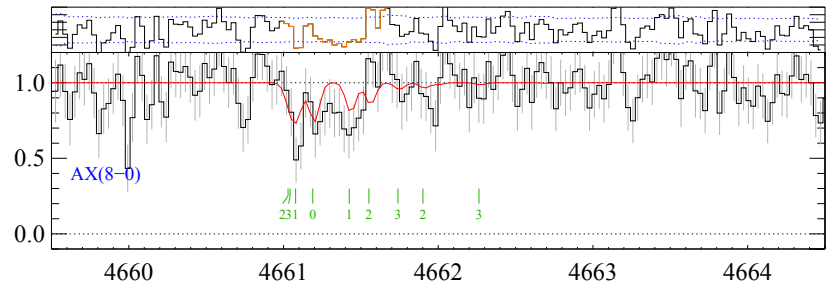

Em

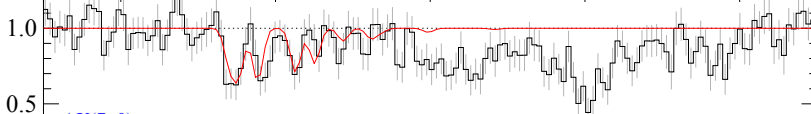
$0.5={ }_{\operatorname{Ax}(7-0)}$
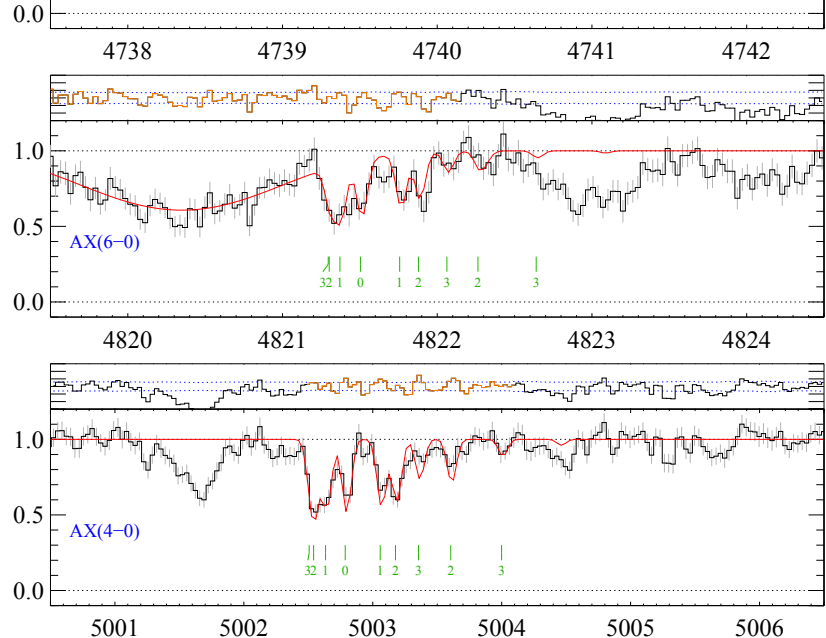

Erring 1.0 EN E

$\begin{array}{llllll}5101 & 5102 & 5103 & 5104 & 5105 & 5106\end{array}$

E 1.0 and $0.0=$ \begin{tabular}{lllllll}
5207 & 5208 & 5209 & 5210 & 5211 & 5212 \\
\hline 5322 & 5323 & 5324 & 5325 & 5326
\end{tabular}

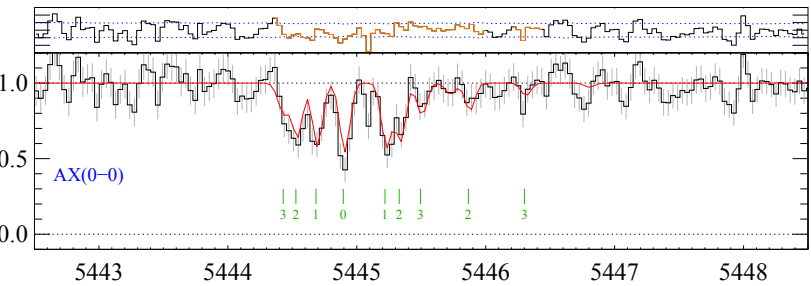

Fig. A.1. Voigt-profile fit to the $\mathrm{CO}$ absorption bands, labelled in blue in each panel. The left (resp. right) panels correspond to data taken with the $0.9^{\prime \prime}$ (resp. $0.7^{\prime \prime}$ ) slit. Rotational levels from $J=0$ to $J=3$ are indicated as green tick marks. The panel above each region shows the residual, with the blue line indicating the $\pm 1 \sigma$ interval, and the orange regions that used to constrain the fit. 

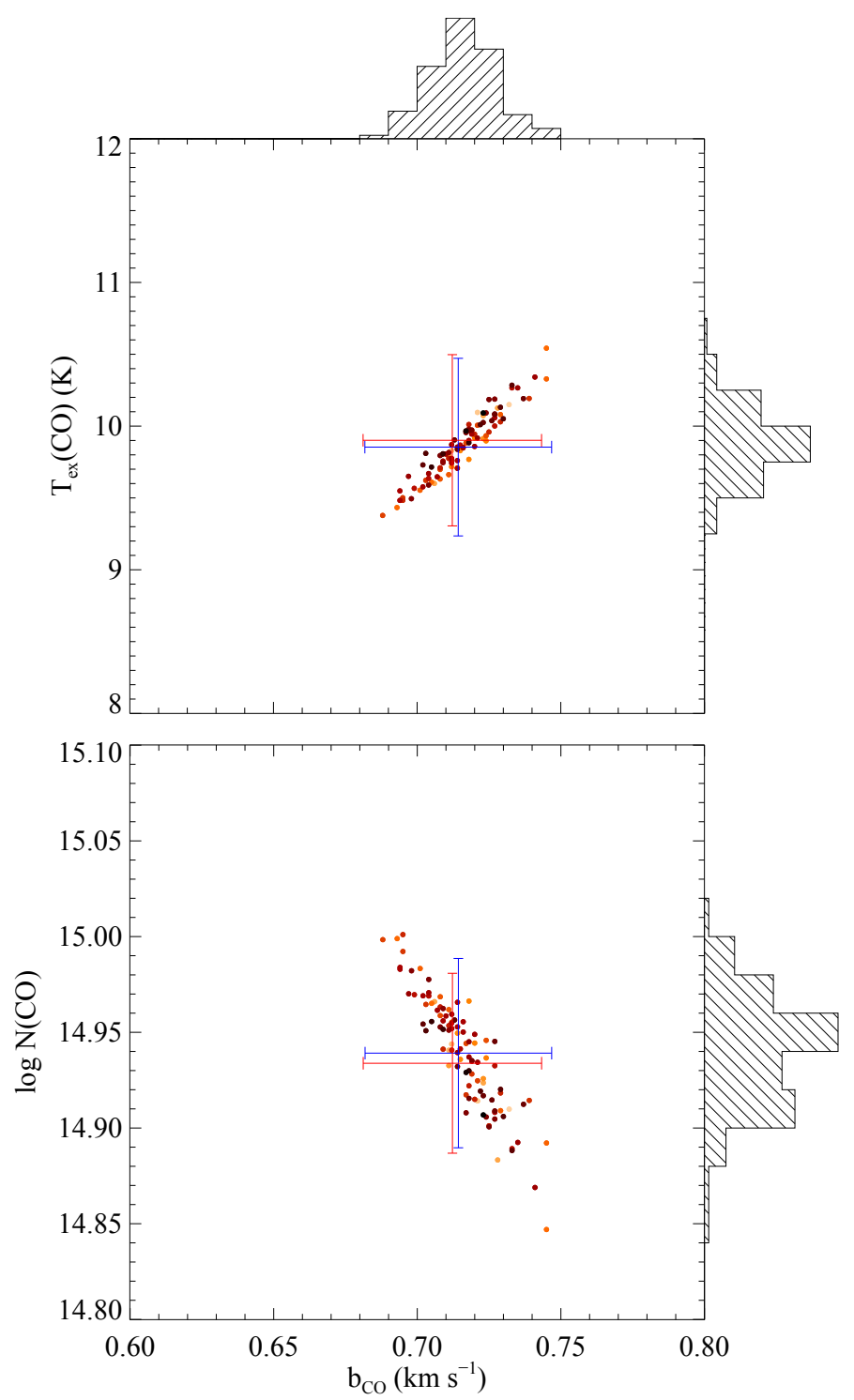

Fig. A.2. Effect of continuum placement uncertainties on the total $\mathrm{CO}$ column density, excitation temperature and Doppler parameter. The histograms represent the distributions of $\log N(\mathrm{CO}), T_{\mathrm{ex}}(\mathrm{CO})$ and $b$ for 100 realisations where the continuum for each fitting region has been modified independently and randomly. The colour of each point indicates the reduced $\chi^{2}$ that varies from 1.08 (darkest) to 1.23 (lightest). The blue (resp. red) error bar corresponds to the best fit value using the combined UVES spectrum (resp. both UVES spectra fitted simultaneously). 